\title{
Synthesis and Characterization of Peralkylated Pyrrole-fused Azacoronene
}

\author{
Kosuke Oki, ${ }^{\dagger}$ Masayoshi Takase,${ }^{\dagger^{*}}$ Nagao Kobayashi, ${ }^{\ddagger^{*}}$ Hidemitsu Uno,,$^{\star *}$ \\ ${ }^{\dagger}$ Graduate School of Science and Engineering, Ehime University, Matsuyama 790-8577, Japan \\ ${ }^{\ddagger}$ Faculty of Textile Science and Technology, Shinshu University, Ueda 386-8567, Japan \\ E-mail: takase.masayoshi.ry@ehime-u.ac.jp \\ nagaok@shinshu-u.ac.jp \\ uno.hidemitsu.mm@ehime-u.ac.jp
}

\section{Contents}

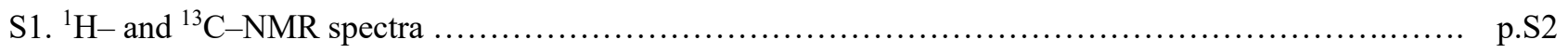

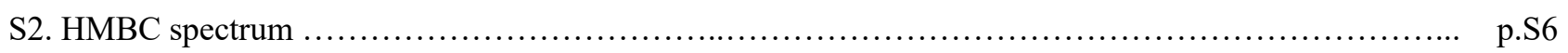

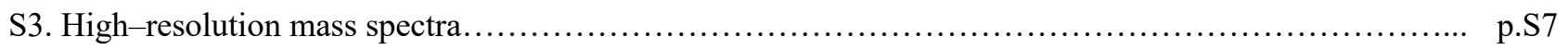

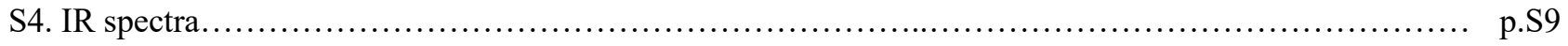

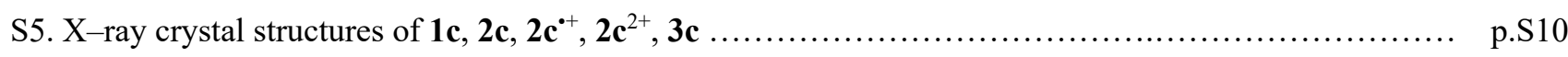

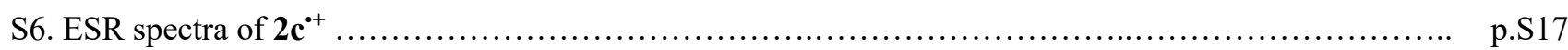

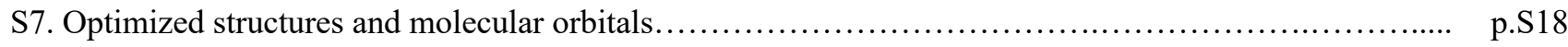

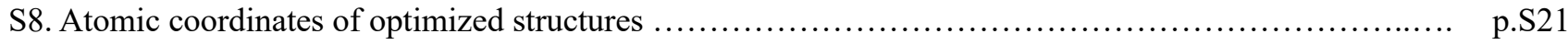

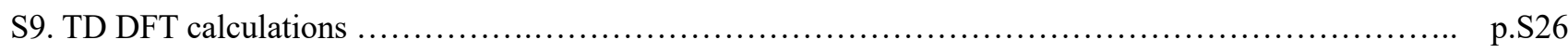

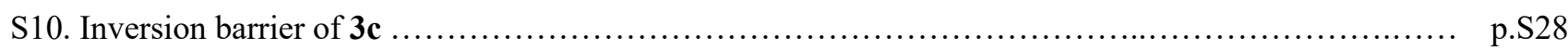

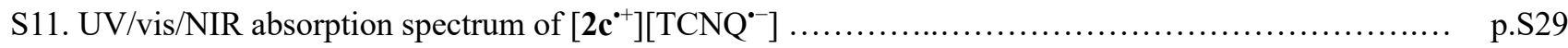

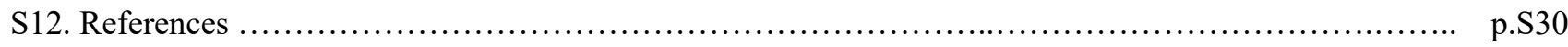


S1. ${ }^{1} \mathrm{H}-$ and ${ }^{13} \mathrm{C}-\mathrm{NMR}$ spectra<smiles>CCc1ccn(-c2c(-n3ccc(CC)c3)c(-n3cc(CC)c(CC)c3)c(-n3cc(CC)cn3)c(-n3cc(CC)cn3)c2-n2cc(CC)c(CC)c2)c1</smiles>

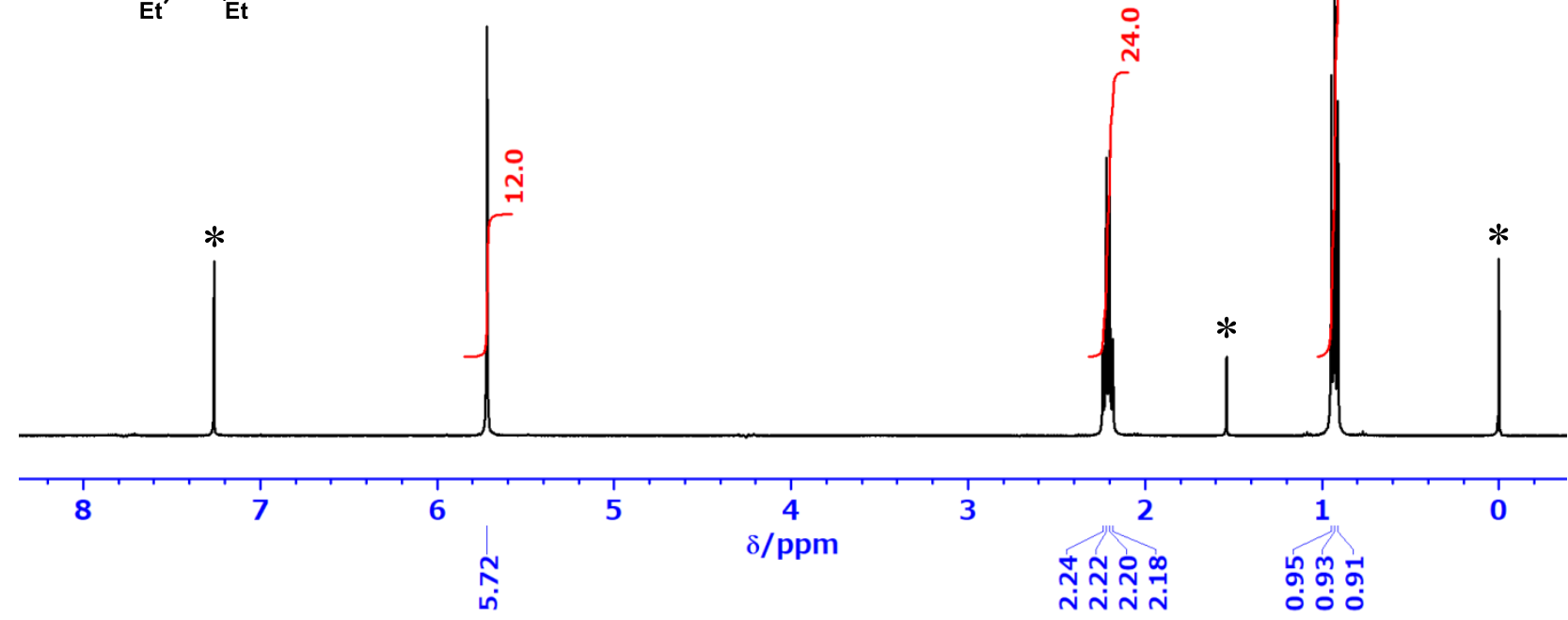

Figure S1a. ${ }^{1} \mathrm{H}-\mathrm{NMR}(400 \mathrm{MHz})$ spectrum of $1 \mathbf{c}$ in $\mathrm{CDCl}_{3}$.

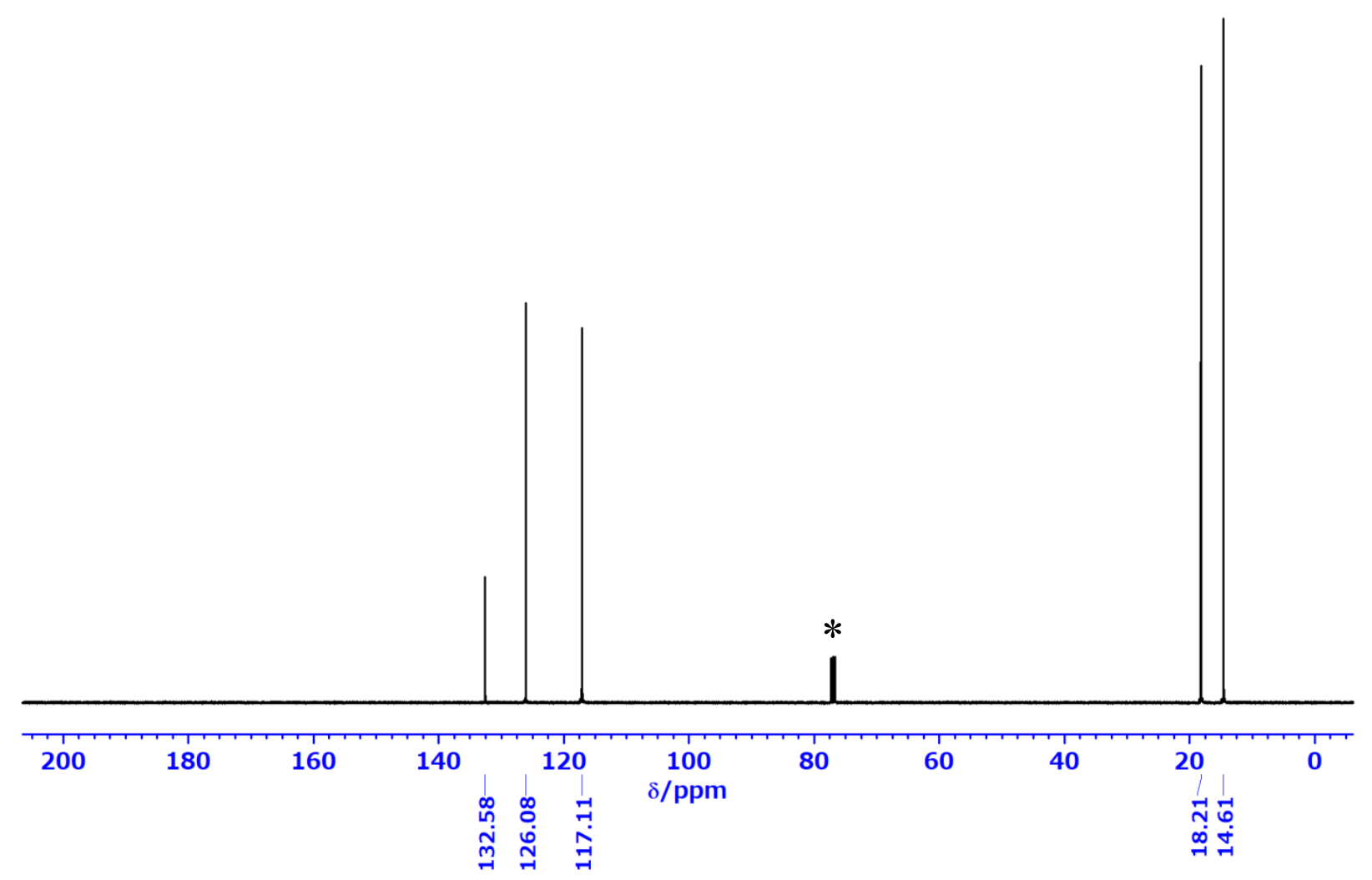

Figure S1b. ${ }^{13} \mathrm{C}\left\{{ }^{1} \mathrm{H}\right\}$-NMR $(100 \mathrm{MHz})$ spectrum of $\mathbf{1 c}$ in $\mathrm{CDCl}_{3}$. 


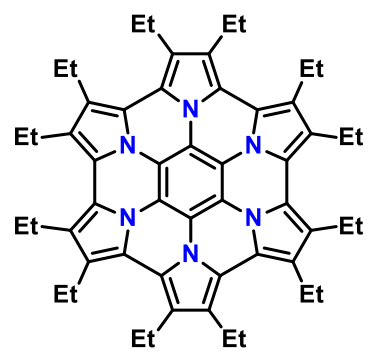

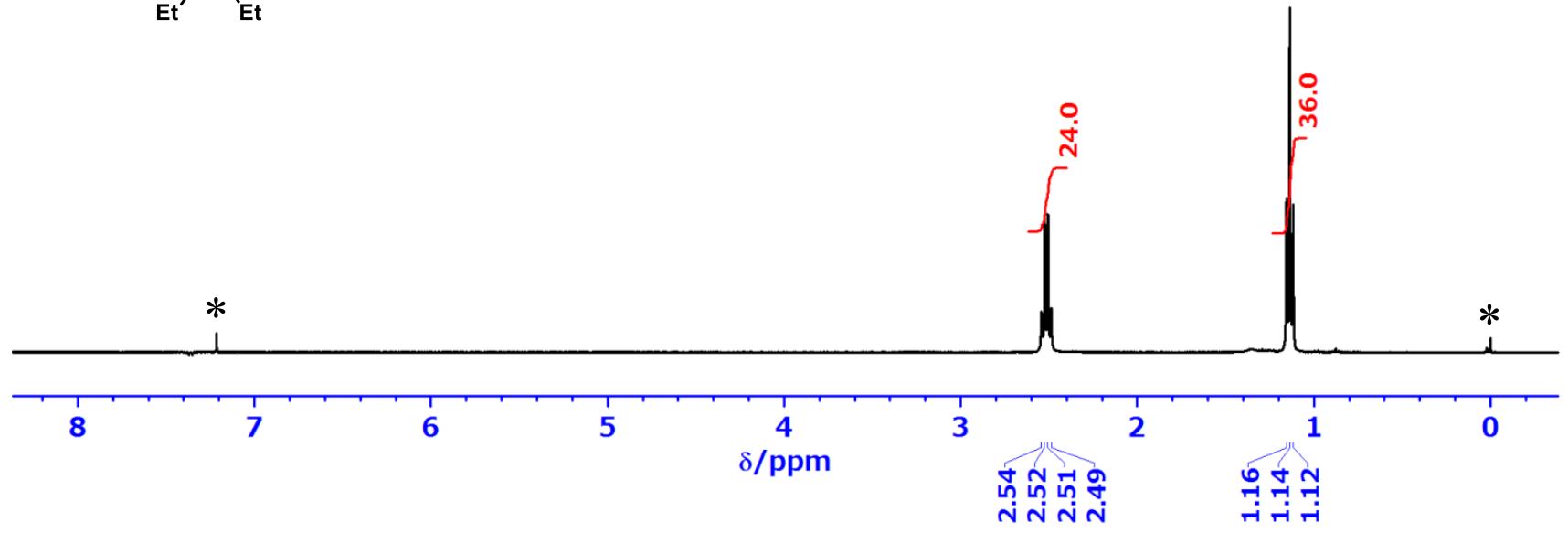

Figure S1c. ${ }^{1} \mathrm{H}-\mathrm{NMR}(400 \mathrm{MHz})$ spectrum of $2 \mathbf{c}$ in a mixed solvent $\left(\mathrm{CDCl}_{3}: \mathrm{CS}_{2}=1: 3\right)$ in the presence of small amount of hydrazine hydrate and $\mathrm{D}_{2} \mathrm{O}$.

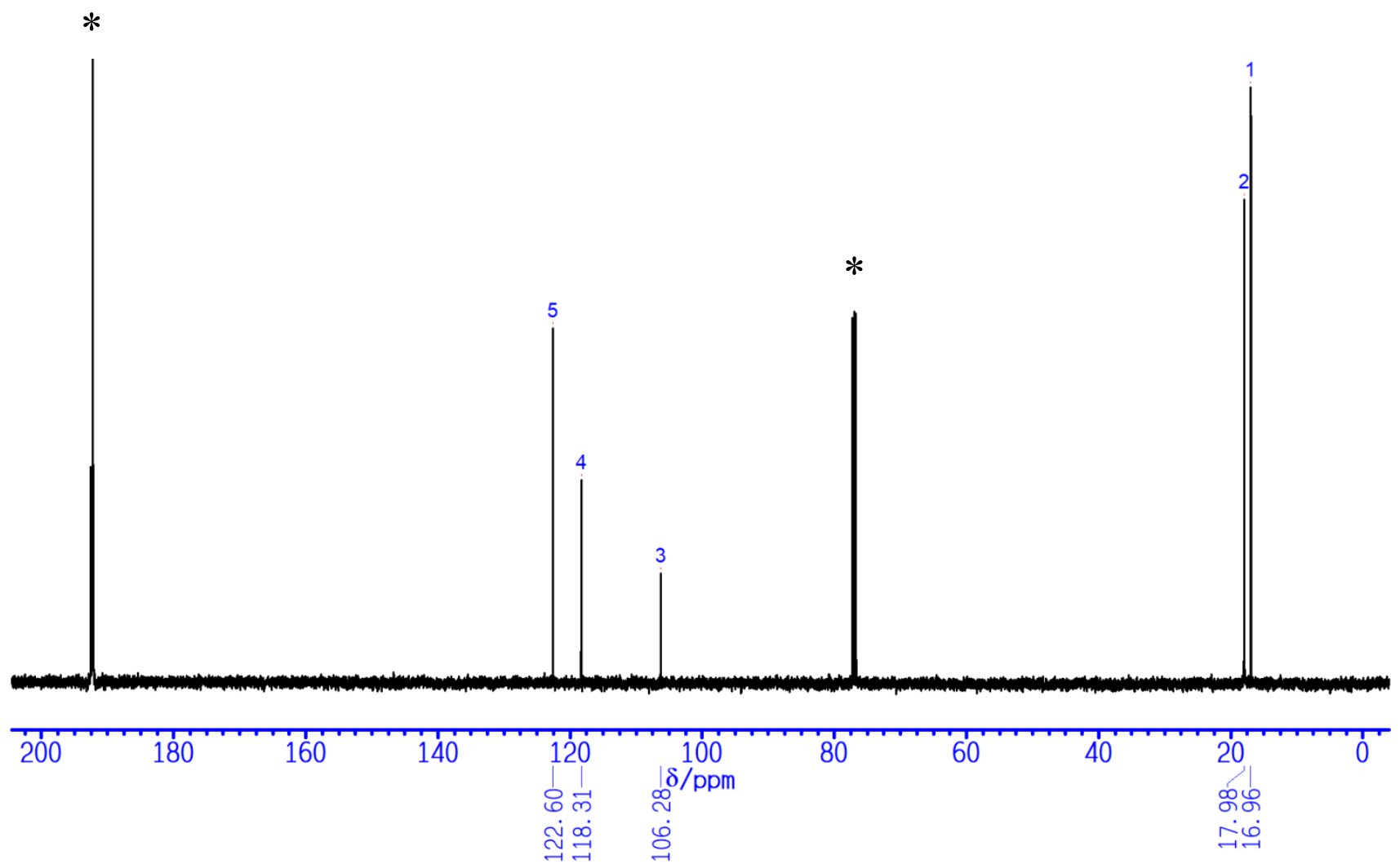

Figure S1d. ${ }^{13} \mathrm{C}\left\{{ }^{1} \mathrm{H}\right\}$-NMR $(100 \mathrm{MHz})$ spectrum of $\mathbf{2 c}$ in a mixed solvent $\left(\mathrm{CDCl}_{3}: \mathrm{CS}_{2}=1: 3\right)$ in the presence of small amount of hydrazine hydrate and $\mathrm{D}_{2} \mathrm{O}$. 


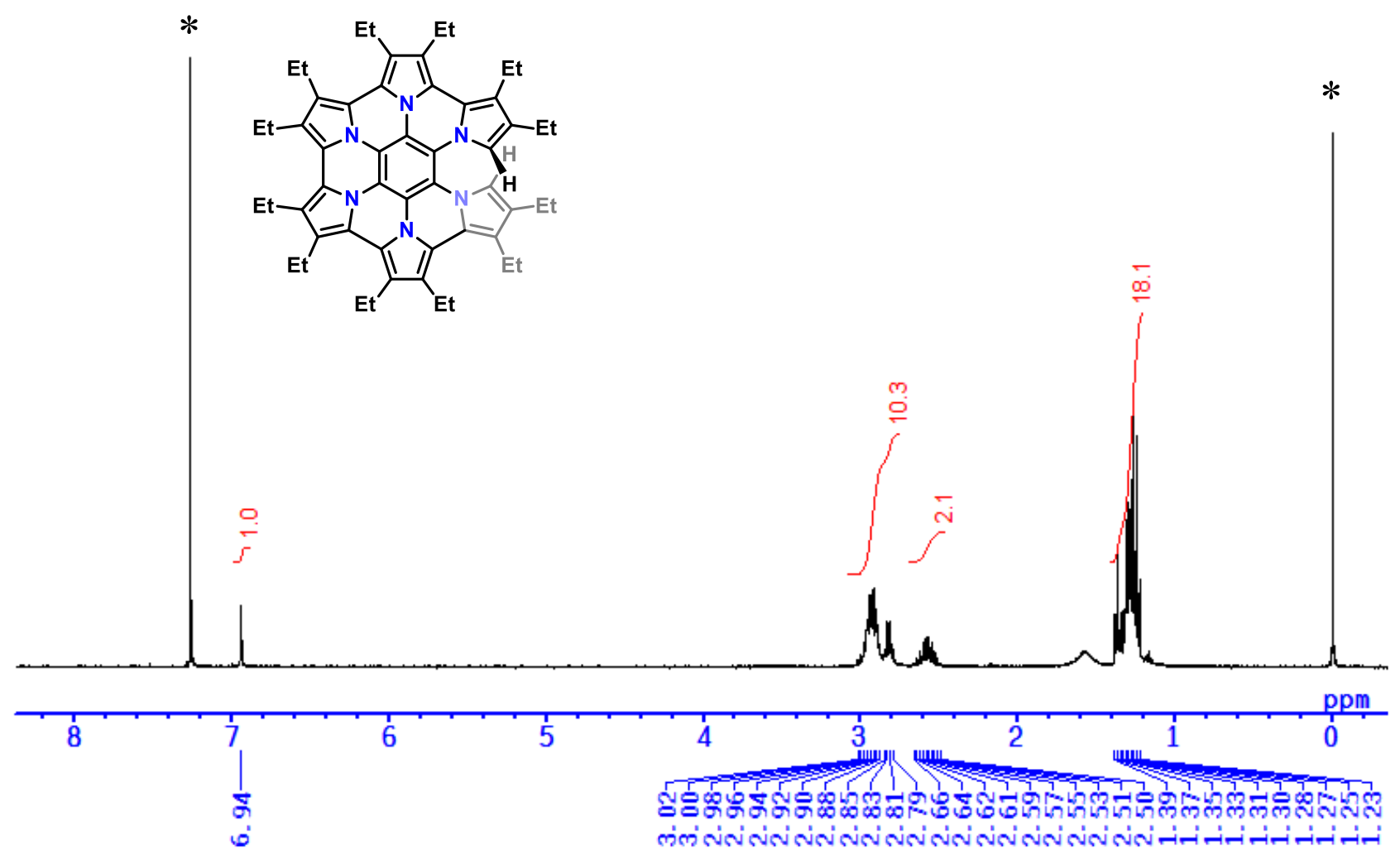

Figure S1e. ${ }^{1} \mathrm{H}-\mathrm{NMR}(400 \mathrm{MHz})$ spectrum of $\mathbf{3 c}$ in $\mathrm{CDCl}_{3}$ in the presence of small amount of hydrazine hydrate and $\mathrm{D}_{2} \mathrm{O}$.

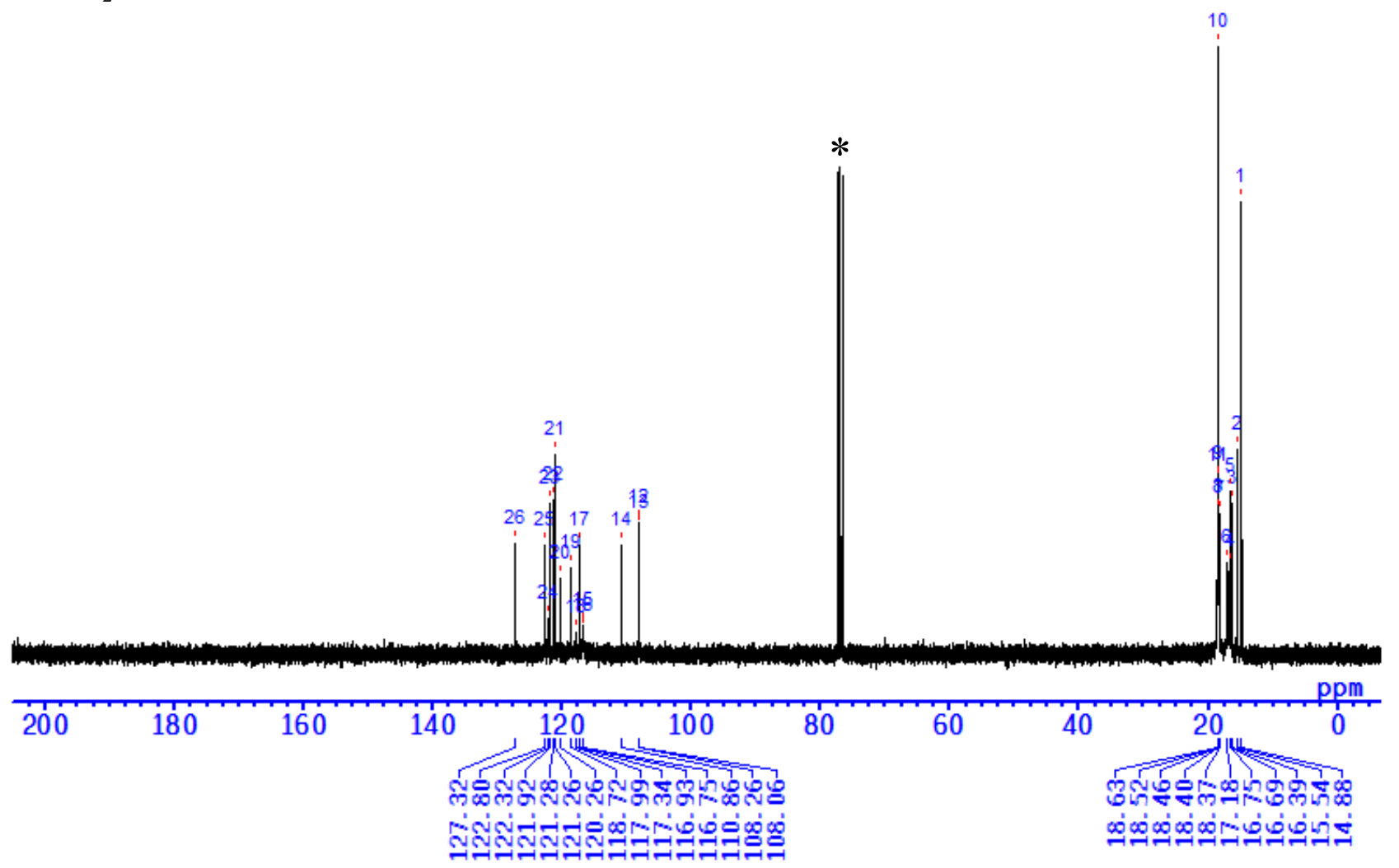

Figure S1f. ${ }^{13} \mathrm{C}\left\{{ }^{1} \mathrm{H}\right\}$-NMR (100 MHz) spectrum of $\mathbf{3 c}$ in $\mathrm{CDCl}_{3}$ in the presence of small amount of hydrazine hydrate and $\mathrm{D}_{2} \mathrm{O}$. 

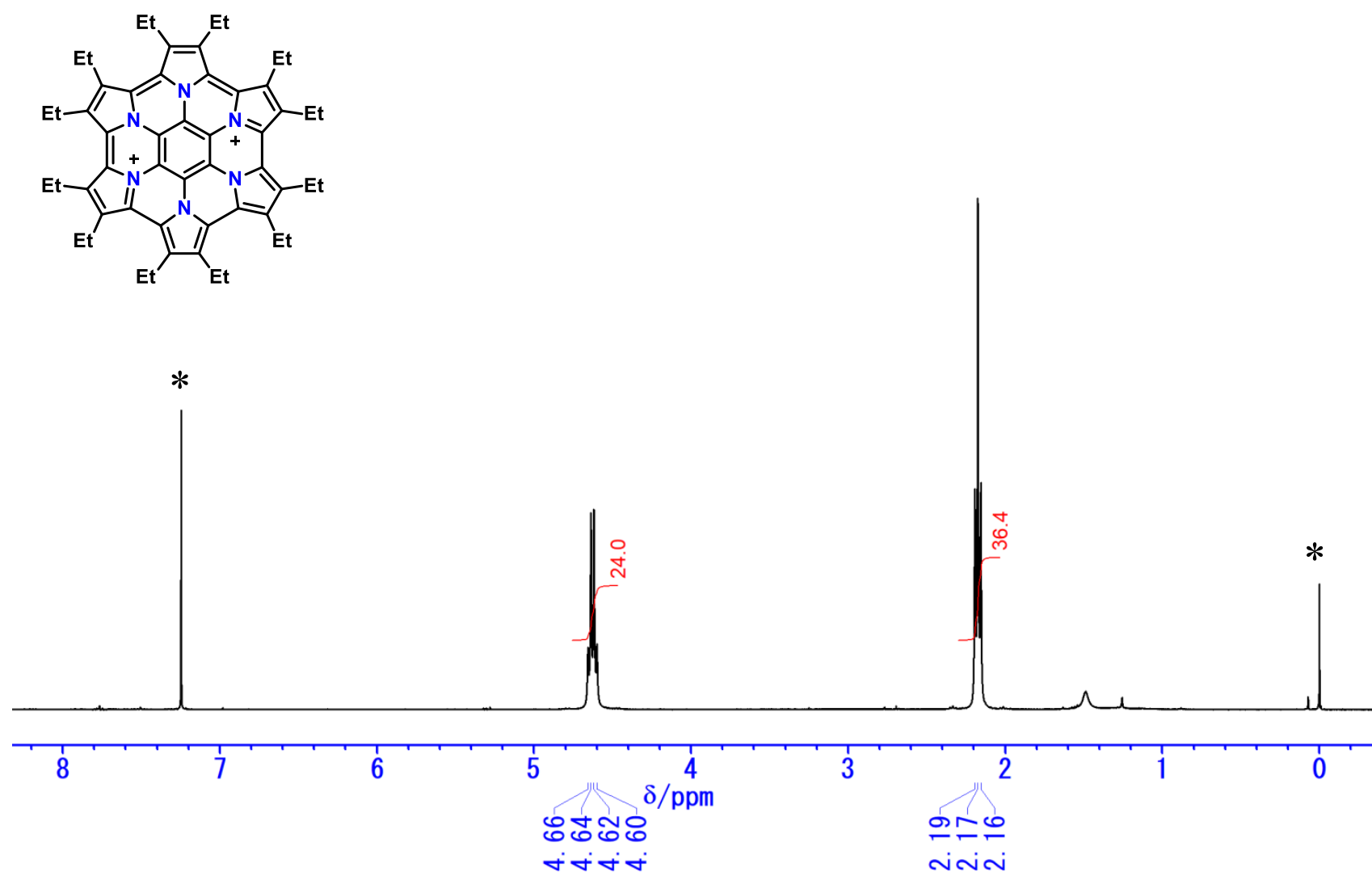

Figure S1g. ${ }^{1} \mathrm{H}-\mathrm{NMR}(400 \mathrm{MHz})$ spectrum of $2 \mathbf{c}^{2+}$ in $\mathrm{CDCl}_{3}$.

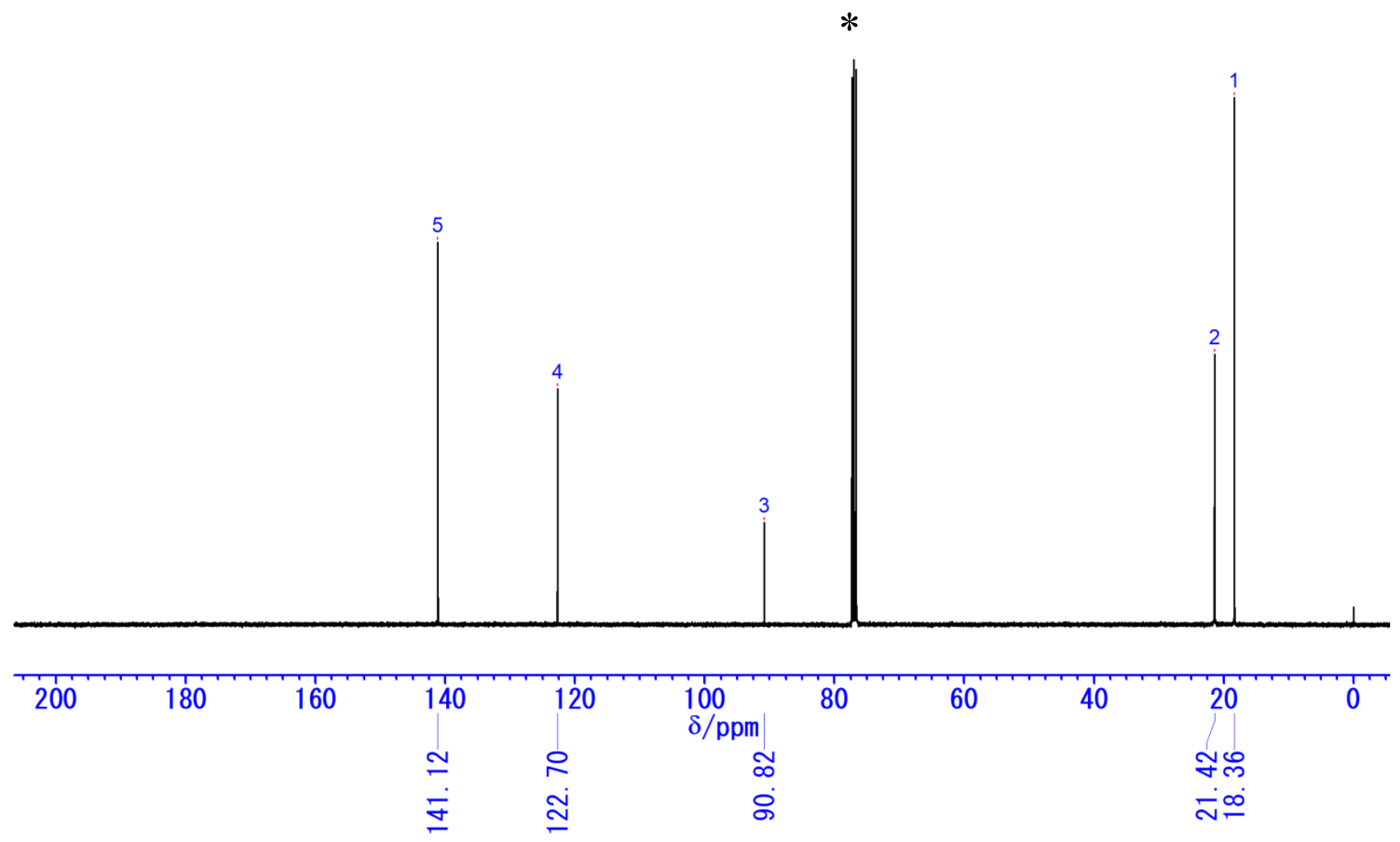

Figure S1h. ${ }^{13} \mathrm{C}\left\{{ }^{1} \mathrm{H}\right\}-\mathrm{NMR}(100 \mathrm{MHz})$ spectrum of $\mathbf{2} \mathbf{c}^{\mathbf{2 +}}$ in $\mathrm{CDCl}_{3}$. 


\section{S2. HMBC spectrum}

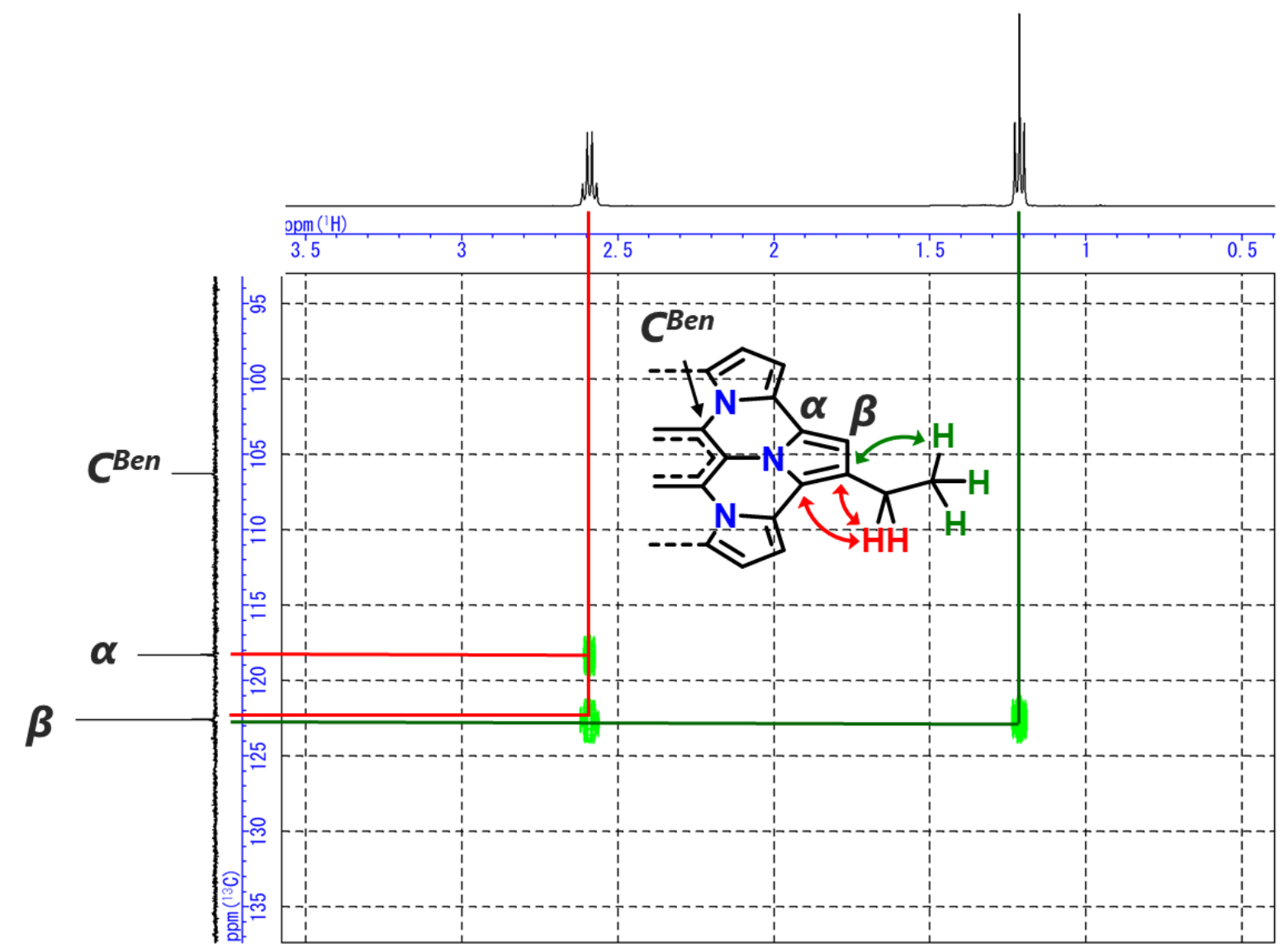

Figure S2. Selected regions of HMBC correlation spectrum of $\mathbf{2} \mathbf{c}$ in a mixed solvent $\left(\mathrm{CDCl}_{3}: \mathrm{CS}_{2}=1: 3\right)$ in the presence of small amount of hydrazine hydrate and $\mathrm{D}_{2} \mathrm{O}$. 


\section{S3. High-resolution mass spectra}

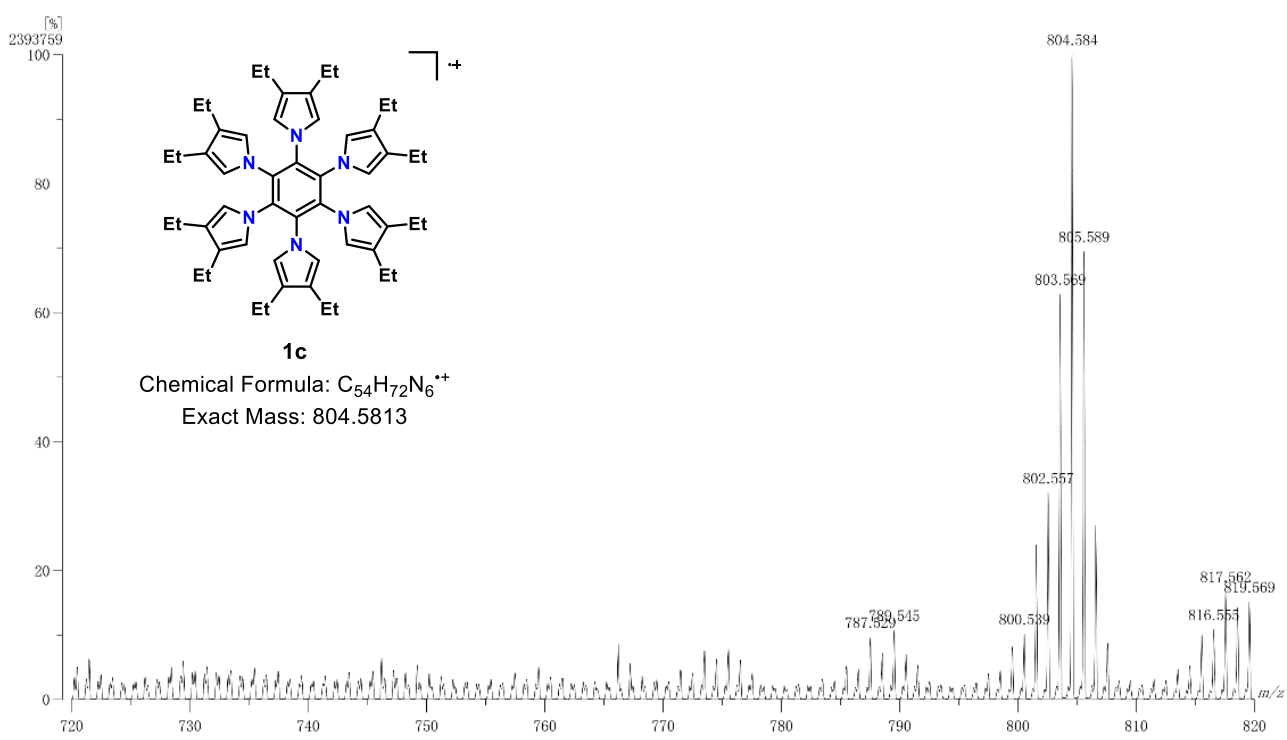

Figure S3a. High-resolution FAB MS spectrum of 1c.

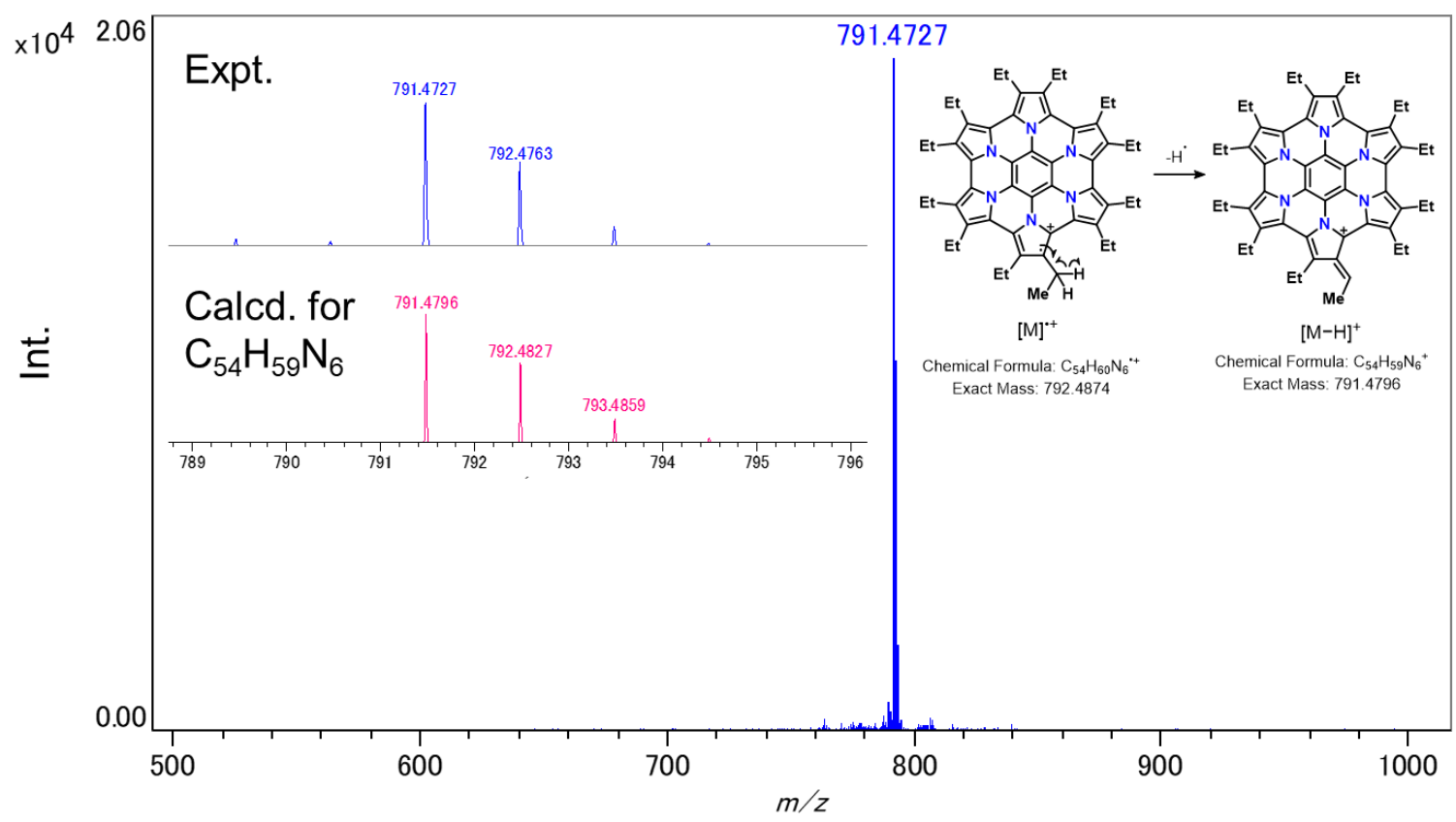

Figure S3b. Positive-ion MALDI-TOF MS spectrum of $\mathbf{2} \mathbf{c}$ using TCNQ as the matrix. The signal corresponding to the cation $[\mathrm{M}-\mathrm{H}]^{+}$was detected probably due to the desorption of hydrogen radical from radical cation of $2 \mathbf{c}\left([\mathrm{M}]^{*+}\right)$. 


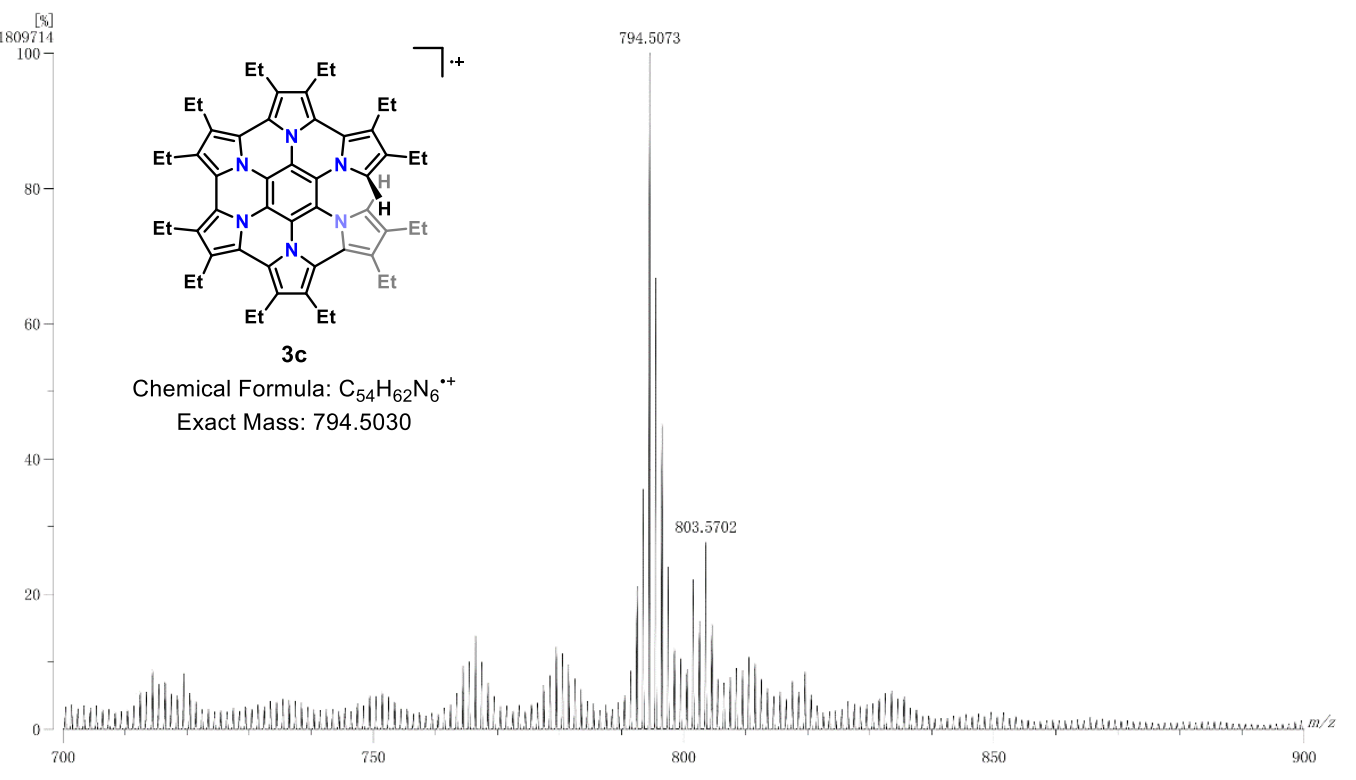

Figure S3c. High-resolution FAB MS spectrum of 3c. 


\section{S4. IR spectra}

(a)

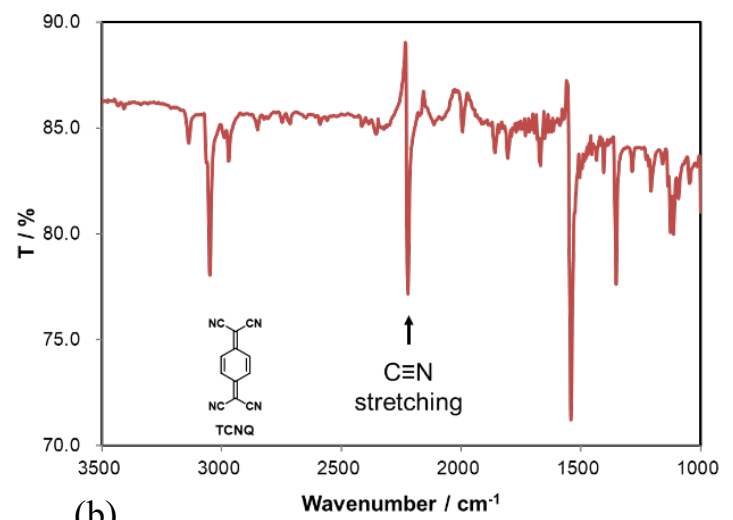

(b)

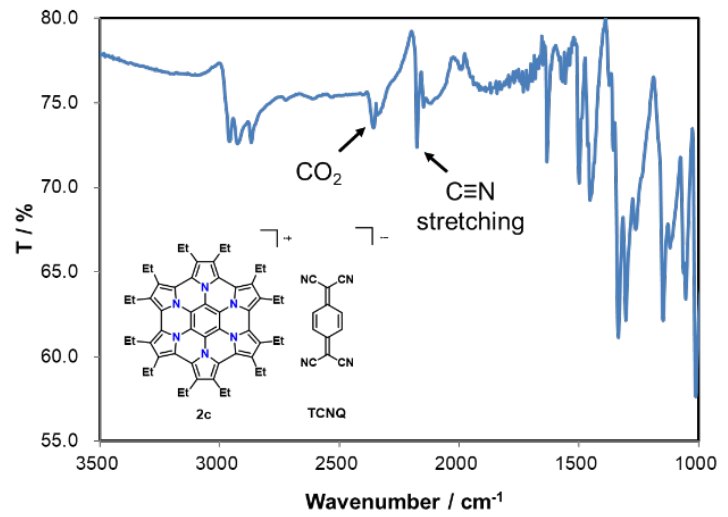

(c)

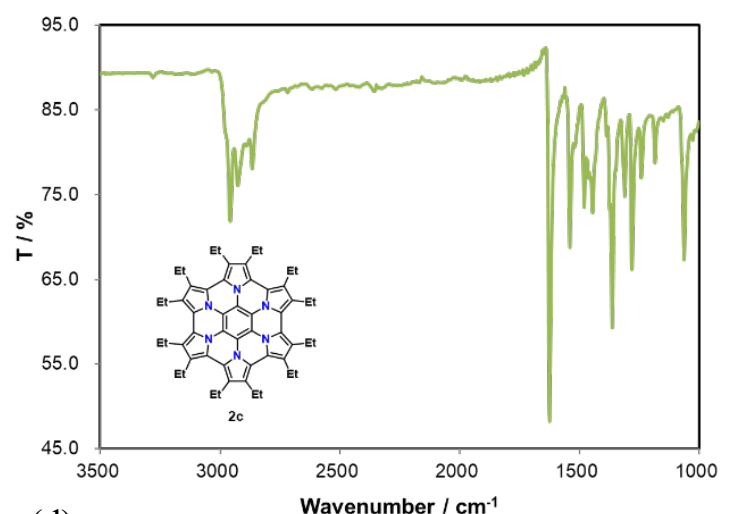

(d)

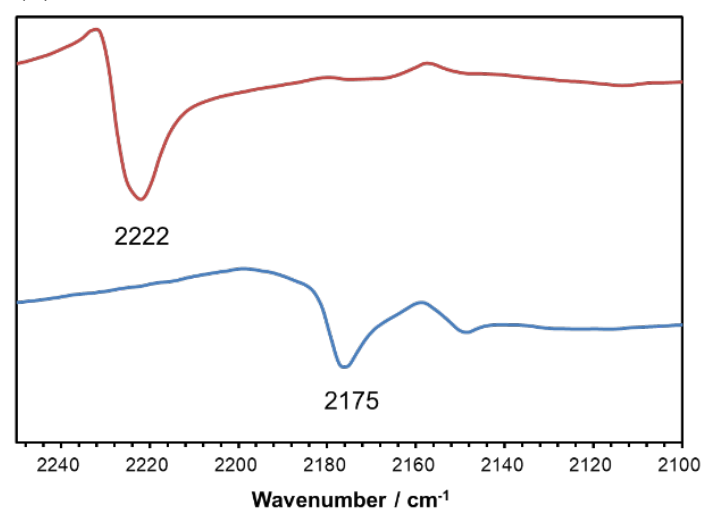

Figure S4. IR spectra of (a) TCNQ, (b) $\left[\mathbf{2 c}^{\mathbf{c}^{++}}\right]\left[\mathrm{TCNQ}^{-}\right]$, and (c) $2 \mathbf{c}$. (d) Comparison of CN stretching between TCNQ and $\left[\mathbf{2}^{\cdot+}\right]\left[\mathrm{TCNQ}^{-}\right]$in the IR spectra. 


\section{S5. X-ray crystal structures}

Crystallographic analysis: X-ray diffraction analysis was performed using a Rigaku VariMax with Saturn diffractometer using multilayer mirror monochromated Mo Ka radiation $(\lambda=0.71073 \AA)$ at $100 \mathrm{~K}$. The crystals were mounted in cryoloops. Data collection was performed using CrystalClear software. Data reduction was performed using CrystalClear or CrysAlisPro software. The data were corrected for Lorentz polarization and absorption effects. The structures were solved using SHELXT 2014/5 $5^{[1]}$ and expanded using the Fourier technique. All calculations were performed using Olex $2^{[2]}$ software. SHELXL-2018/3 ${ }^{[3]}$ was used for structure refinement. The data were validated using PLATON.

Table S5a. Crystallographic refinement data for 1c and 2c.

\begin{tabular}{|c|c|c|}
\hline & 1c & $2 c$ \\
\hline Formula & $\mathrm{C}_{54} \mathrm{H}_{72} \mathrm{~N}_{6}$ & $\mathrm{C}_{54} \mathrm{H}_{60} \mathrm{~N}_{6}$ \\
\hline Formula weight & 805.17 & 793.08 \\
\hline Size $[\mathrm{mm}]$ & $0.30 \times 0.25 \times 0.15$ & $0.10 \times 0.08 \times 0.02$ \\
\hline Radiation & $\operatorname{Mo} K \alpha$ & $\operatorname{Mo} K \alpha$ \\
\hline Temperature $[\mathrm{K}]$ & 100 & 100 \\
\hline Crystal system & monoclinic & triclinic \\
\hline Space group & $P 2_{1} / c(\# 14)$ & $P-1(\# 2)$ \\
\hline$a[\AA]$ & $28.558(4)$ & $9.2171(3)$ \\
\hline$b[\AA]$ & $20.711(3)$ & $14.7522(5)$ \\
\hline$c[\AA]$ & $16.320(2)$ & $15.8309(6)$ \\
\hline$\alpha\left[^{\circ}\right]$ & 90 & $76.939(3)$ \\
\hline$\beta\left[^{\circ}\right]$ & $94.231(2)$ & $78.747(3)$ \\
\hline$\gamma\left[^{\circ}\right]$ & 90 & $89.752(3)$ \\
\hline$V\left[\AA^{3}\right]$ & $9626(2)$ & $2054.79(13)$ \\
\hline $\mathrm{Z}$ & 8 & 2 \\
\hline$\rho\left[\mathrm{g} \cdot \mathrm{cm}^{-3}\right]$ & 1.111 & 1.282 \\
\hline$\mu\left[\mathrm{cm}^{-1}\right]$ & 0.651 & 0.755 \\
\hline $\mathrm{F}(000)$ & 3504.00 & 852.00 \\
\hline \multirow[t]{2}{*}{ No. of reflections measured } & 166677 (Total) & 33961 (Total) \\
\hline & 23791 (Unique) & 9420 (Unique) \\
\hline$R_{\text {int }}$ & 0.1202 & 0.0475 \\
\hline$R_{1}$ & 0.0952 & 0.0580 \\
\hline$w R_{2}$ & 0.2318 & 0.1506 \\
\hline GOF & 1.167 & 1.017 \\
\hline CCDC No. & 2051723 & 2051724 \\
\hline
\end{tabular}


Table S5b. Crystallographic refinement data for $\mathbf{2} \mathbf{c}^{\mathbf{}+}$.

\begin{tabular}{|c|c|c|}
\hline & {$\left[2 \mathbf{c}^{\cdot+}\right]\left[\mathrm{SbF}_{6}^{-}\right]$} & {$\left[\mathbf{2}^{\cdot+}\right]\left[\mathrm{TCNQ}^{-}\right]$} \\
\hline Formula & $\mathrm{C}_{54} \mathrm{H}_{60} \mathrm{~N}_{6}, \mathrm{C}_{6} \mathrm{H}_{5} \mathrm{Cl}, \mathrm{SbF}_{6}$ & $\mathrm{C}_{54} \mathrm{H}_{60} \mathrm{~N}_{6}, \mathrm{C}_{12} \mathrm{H}_{4} \mathrm{~N}_{4}, 3\left(\mathrm{C}_{2} \mathrm{H}_{2} \mathrm{Cl}_{4}\right)$ \\
\hline Formula weight & 1141.41 & 1500.78 \\
\hline Size $[\mathrm{mm}]$ & $0.20 \times 0.15 \times 0.12$ & $0.20 \times 0.07 \times 0.04$ \\
\hline Radiation & $\operatorname{Mo} K \alpha$ & $\operatorname{MoK} \alpha$ \\
\hline Temperature $[\mathrm{K}]$ & 100 & 100 \\
\hline Crystal system & trigonal & orthorhombic \\
\hline Space group & $R-3 c(\# 167)$ & Cmcm (\#63) \\
\hline$a[\AA]$ & $16.0397(3)$ & $30.9781(8)$ \\
\hline$b[\AA]$ & $16.0397(3)$ & $16.1869(4)$ \\
\hline$c[\AA]$ & $71.9620(14)$ & $13.8604(4)$ \\
\hline$\alpha\left[^{\circ}\right]$ & 90 & 90 \\
\hline$\beta\left[^{\circ}\right]$ & 90 & 90 \\
\hline$\gamma\left[^{\circ}\right]$ & 120 & 90 \\
\hline$V\left[\AA^{3}\right]$ & $16033.4(5)$ & $6950.2(3)$ \\
\hline $\mathrm{Z}$ & 12 & 4 \\
\hline$\rho\left[\mathrm{g} \cdot \mathrm{cm}^{-3}\right]$ & 1.418 & 1.434 \\
\hline$\mu\left[\mathrm{cm}^{-1}\right]$ & 6.320 & 5.290 \\
\hline $\mathrm{F}(000)$ & 7068.00 & 3104.00 \\
\hline \multirow[t]{2}{*}{ No. of reflections measured } & 82027 (Total) & 57236 (Total) \\
\hline & 4097 (Unique) & 4229 (Unique) \\
\hline$R_{\text {int }}$ & 0.0373 & 0.0693 \\
\hline$R_{1}$ & 0.0688 & 0.0527 \\
\hline$w R_{2}$ & 0.2269 & 0.1252 \\
\hline GOF & 1.032 & 1.050 \\
\hline CCDC No. & 2051725 & 2051726 \\
\hline
\end{tabular}


Table S5c. Crystallographic refinement data for $\mathbf{2} \mathrm{c}^{2+}$.

\begin{tabular}{|c|c|c|}
\hline & {$\left[2 \mathbf{c}^{2+}\right]\left[\mathrm{SbF}_{6}^{-}\right]_{2}$} & {$\left[2 \mathbf{c}^{2+}\right]\left[\mathrm{I}_{3}{ }^{-}\right]_{2}$} \\
\hline Formula & $\mathrm{C}_{54} \mathrm{H}_{60} \mathrm{~N}_{6}, 3 \mathrm{CHCl}_{3}, 2 \mathrm{SbF}_{6}$ & $\mathrm{C}_{54} \mathrm{H}_{60} \mathrm{~N}_{6}, 2\left(\mathrm{I}_{3}\right)$ \\
\hline Formula weight & 1622.68 & 1554.48 \\
\hline Size $[\mathrm{mm}]$ & $0.12 \times 0.08 \times 0.03$ & $0.12 \times 0.04 \times 0.02$ \\
\hline Radiation & $\operatorname{Mo} K \alpha$ & $\operatorname{Mo} K \alpha$ \\
\hline Temperature $[\mathrm{K}]$ & 100 & 100 \\
\hline Crystal system & monoclinic & triclinic \\
\hline Space group & $P 2{ }_{1} / n(\# 14)$ & $P-1(\# 2)$ \\
\hline$a[\AA]$ & $10.8254(3)$ & $9.3231(3)$ \\
\hline$b[\AA]$ & $18.6871(4)$ & $10.3087(3)$ \\
\hline$c[\AA]$ & $16.4606(4)$ & $14.2551(5)$ \\
\hline$\alpha\left[^{\circ}\right]$ & 90 & $100.677(3)$ \\
\hline$\beta\left[^{\circ}\right]$ & $103.727(3)$ & $97.577(3)$ \\
\hline$\gamma\left[^{\circ}\right]$ & 90 & $99.767(2)$ \\
\hline$V\left[\AA^{3}\right]$ & $3234.79(14)$ & $1307.81(8)$ \\
\hline Z & 2 & 1 \\
\hline$\rho\left[\mathrm{g} \cdot \mathrm{cm}^{-3}\right]$ & 1.666 & 1.974 \\
\hline$\mu\left[\mathrm{cm}^{-1}\right]$ & 12.825 & 36.071 \\
\hline $\mathrm{F}(000)$ & 1620.00 & 744.00 \\
\hline \multirow[t]{2}{*}{ No. of reflections measured } & 53228 (Total) & 21132 (Total) \\
\hline & 7416 (Unique) & 6000 (Unique) \\
\hline$R_{i n t}$ & 0.0571 & 0.0436 \\
\hline$R_{1}$ & 0.0344 & 0.0328 \\
\hline$w R_{2}$ & 0.0792 & 0.0684 \\
\hline GOF & 1.031 & 1.027 \\
\hline CCDC No. & 2051727 & 2051728 \\
\hline
\end{tabular}


Table S5d. Crystallographic refinement data for 3c.

\begin{tabular}{|c|c|}
\hline & $3 c$ \\
\hline Formula & $\mathrm{C}_{54} \mathrm{H}_{61.74} \mathrm{~N}_{6}, \mathrm{C}_{2} \mathrm{H}_{2} \mathrm{Cl}_{4}$ \\
\hline Formula weight & 960.91 \\
\hline Size $[\mathrm{mm}]$ & $0.18 \times 0.10 \times 0.04$ \\
\hline Radiation & $\operatorname{MoK} \alpha$ \\
\hline Temperature $[\mathrm{K}]$ & 100 \\
\hline Crystal system & monoclinic \\
\hline Space group & $C 2 / c(\# 15)$ \\
\hline$a[\AA]$ & $15.8290(12)$ \\
\hline$b[\AA]$ & $23.9175(17)$ \\
\hline$c[\AA]$ & $12.8865(10)$ \\
\hline$\alpha\left[^{\circ}\right]$ & 90 \\
\hline$\beta\left[^{\circ}\right]$ & $102.197(7)$ \\
\hline$\gamma\left[^{\circ}\right]$ & 90 \\
\hline$V\left[\AA^{3}\right]$ & $4768.6(6)$ \\
\hline $\mathrm{Z}$ & 4 \\
\hline$\rho\left[\mathrm{g} \cdot \mathrm{cm}^{-3}\right]$ & 1.338 \\
\hline$\mu\left[\mathrm{cm}^{-1}\right]$ & 26.06 \\
\hline $\mathrm{F}(000)$ & 2032.0 \\
\hline \multirow[t]{2}{*}{ No. of reflections measured } & 27828 (Total) \\
\hline & 4316 (Unique) \\
\hline$R_{\text {int }}$ & 0.1007 \\
\hline$R_{l}$ & 0.1106 \\
\hline$w R_{2}$ & 0.2457 \\
\hline GOF & 1.168 \\
\hline CCDC No. & 2051729 \\
\hline
\end{tabular}


(a)

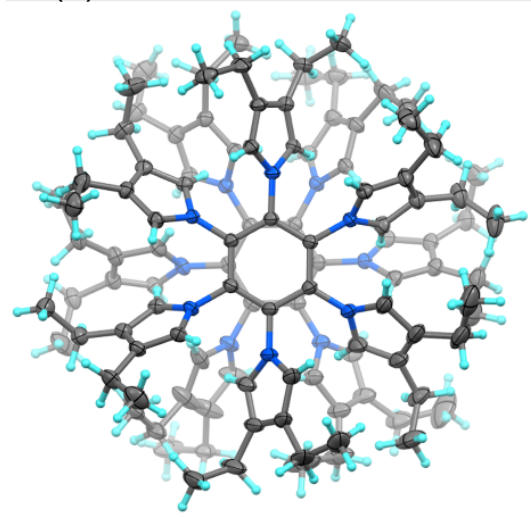

(b)
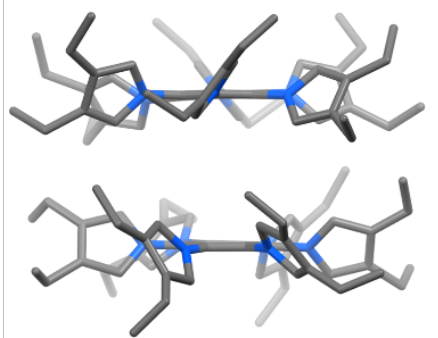

(c)

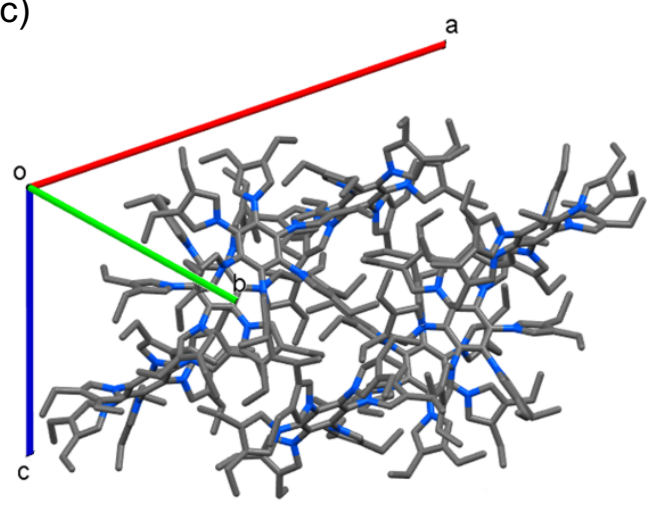

Figure S5a. (a) Top, (b) side, and (c) packing view of $1 \mathbf{c}$.

(a)

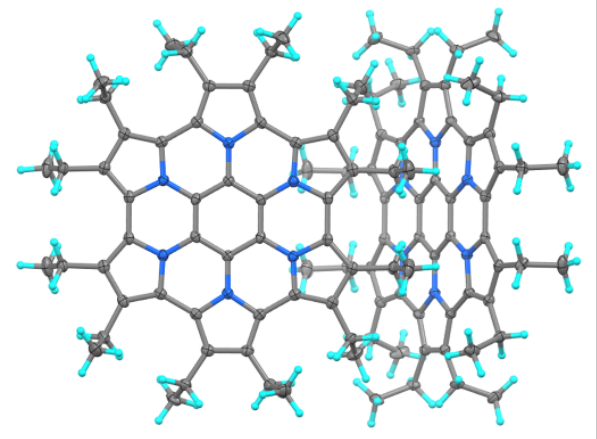

(b)

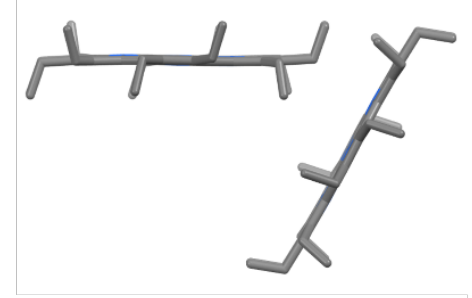

(c)

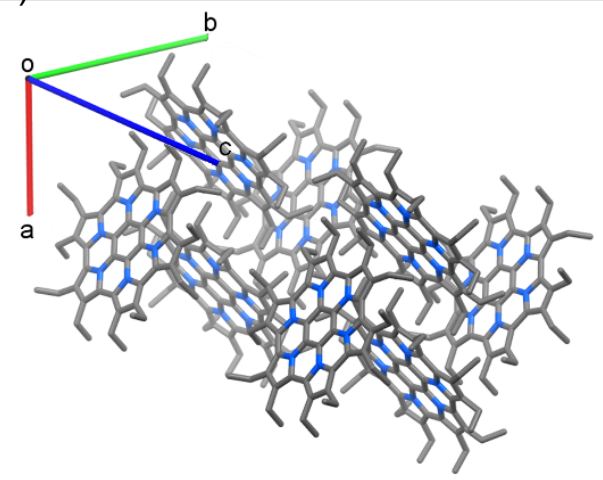

Figure S5b. (a) Top, (b) side, and (c) packing view of 2c.

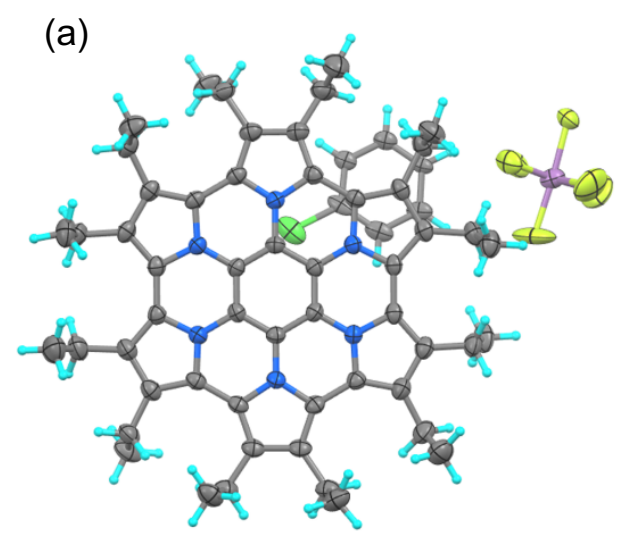

(b)

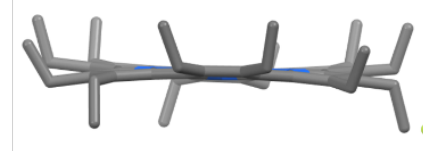

(c)

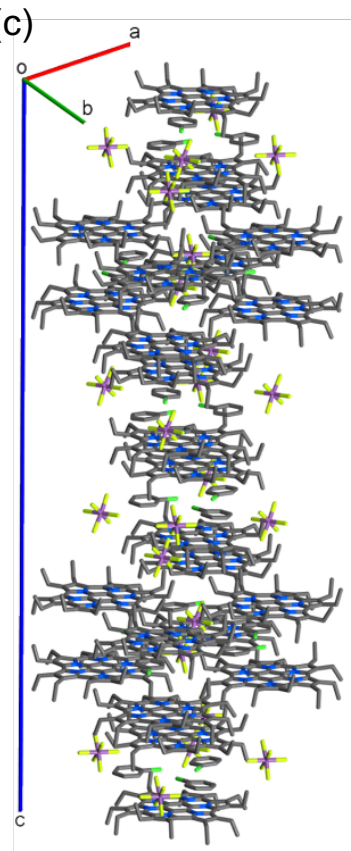

Figure S5c. (a) Top, (b) side, and (c) packing view of $\left[\mathbf{2 c}^{\cdot+}\right]\left[\mathrm{SbF}_{6}{ }^{-}\right]$. 
(a)

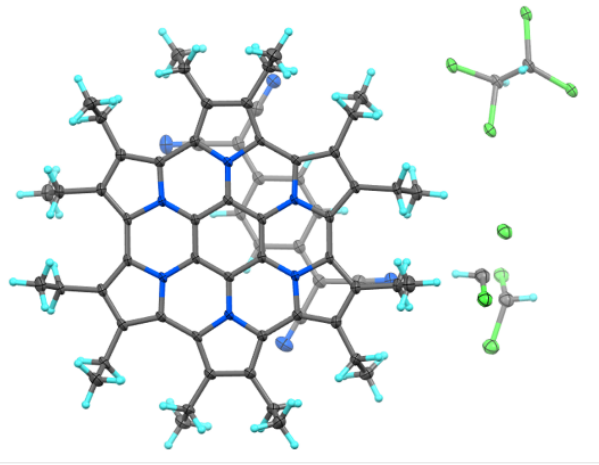

(b)

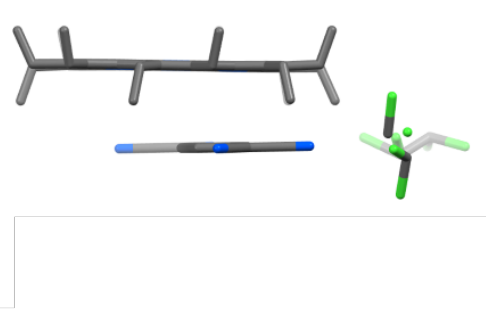

(c)

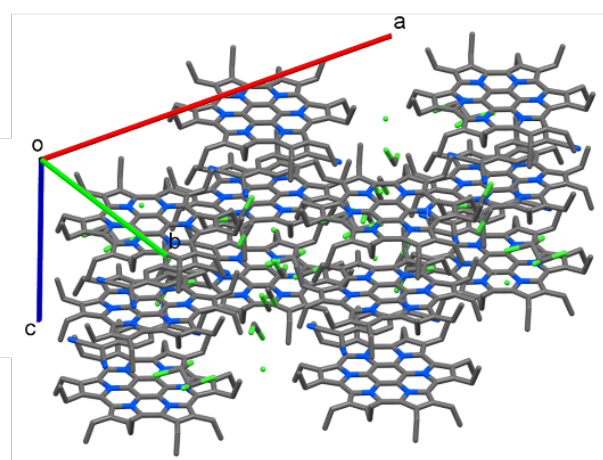

Figure S5d. (a) Top, (b) side, and (c) packing view of [ $\left.\mathbf{2 c}^{\mathbf{c}^{+}}\right]\left[\mathrm{TCNQ}^{\circ}\right]$.

(a)

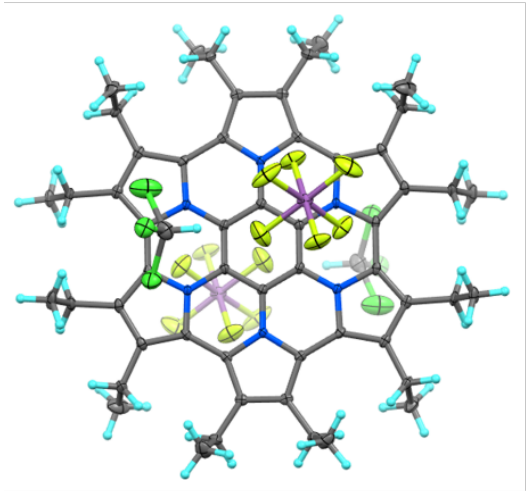

(b)

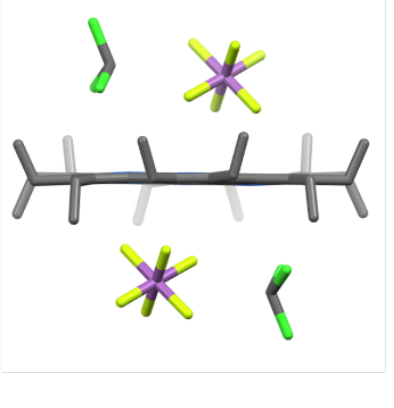

(c)

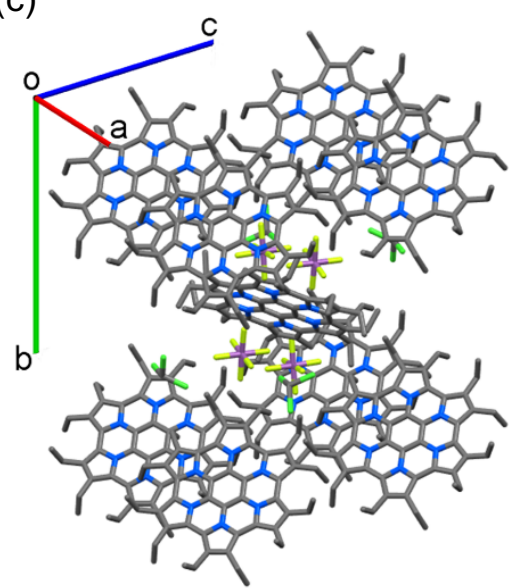

Figure S5e. (a) Top, (b) side, and (c) packing view of $\left[\mathbf{2 c}^{2+}\right]\left[\mathrm{SbF}_{6}^{-}\right]_{2}$.

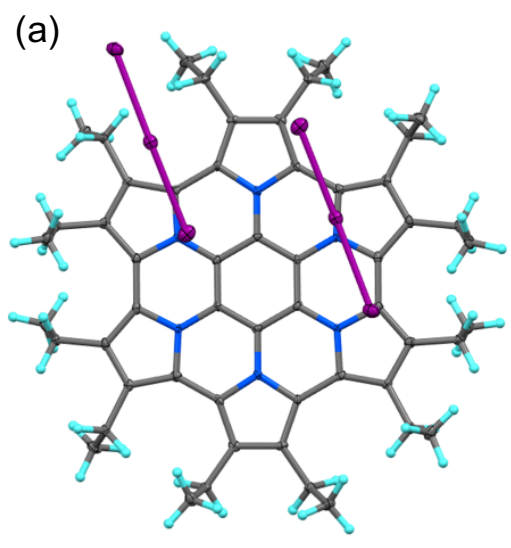

(b)

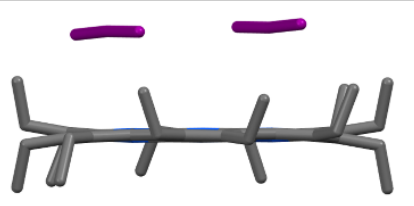

Figure S5f. (a) Top, (b) side, and (c) packing view of $\left[\mathbf{2 c}^{2+}\right]\left[\mathrm{I}_{3}{ }^{-}\right]_{2}$.

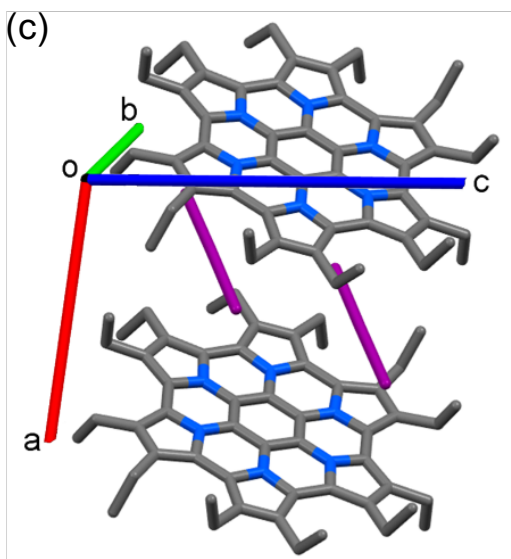


(a)

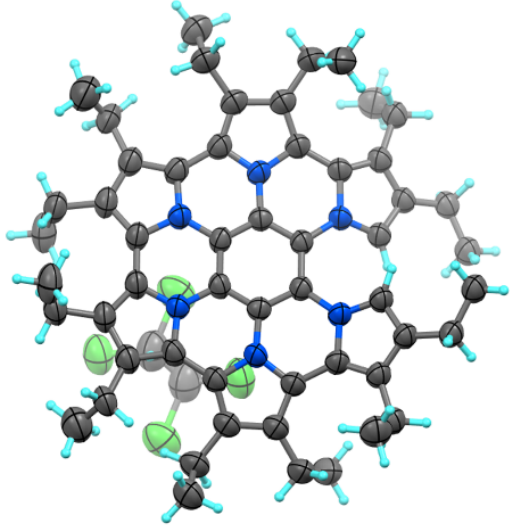

(b)

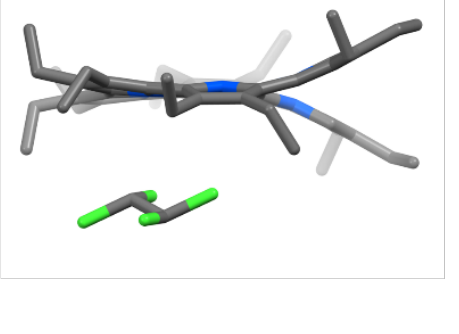

(c)

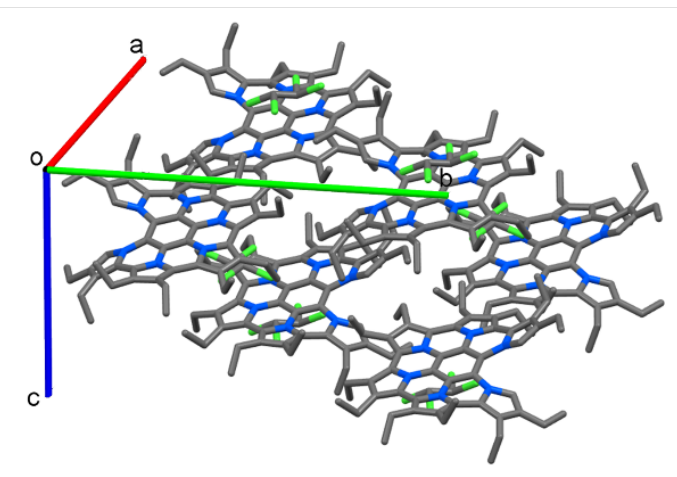

Figure S5g. (a) Top, (b) side, and (c) packing view of 3c. 
S6. ESR spectra

(a)

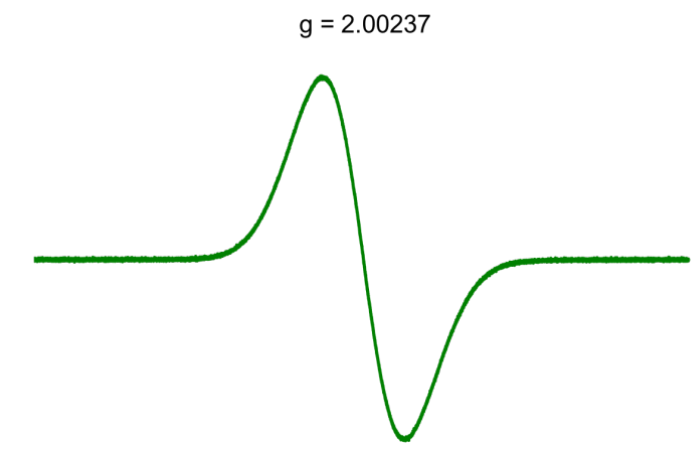

$\begin{array}{lllllll}327.9 & 328.4 & 328.9 & 329.4 & 329.9 & 330.4 & 330.9\end{array}$

Magnetic field $/ \mathrm{mT} \longrightarrow$ (b)

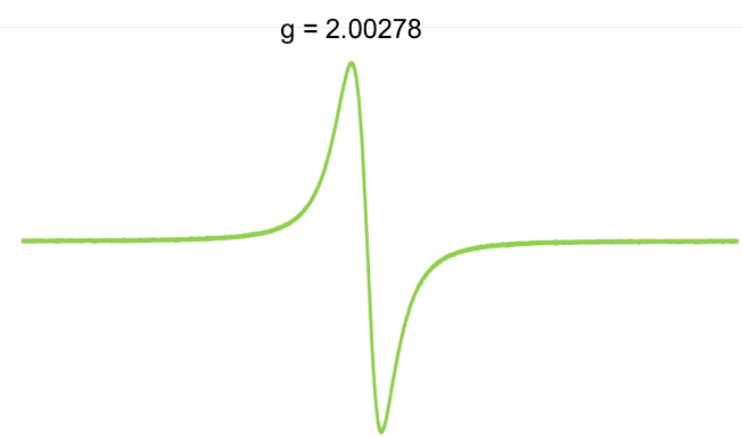

328
329

Magnetic field / $\mathrm{mT} \longrightarrow$

Figure S6. ESR spectra of (a) $\left[2 \mathbf{c}^{*+}\right]\left[\mathrm{SbF}_{6}^{-}\right]$in $\mathrm{CH}_{2} \mathrm{Cl}_{2}$ and (b) $\left[\mathbf{2} \mathbf{c}^{-+}\right]\left[\mathrm{TCNQ}^{*}\right]$ in the crystal. 


\section{S7. Optimized structures and molecular orbitals}

The GAUSSIAN $09^{[4]}$ series of programs was used for all calculations. All molecules were fully optimized using the hybrid density functional at the B3LYP level of theory with the 6-31G (d) basis set. Frequency calculations were conducted to ensure that these structures were indeed local minima Thus, the numbers of imaginary frequencies are 0 for all the optimized structures. Visualization of the results was performed by use of Gauss View 5.0 software.

(a)
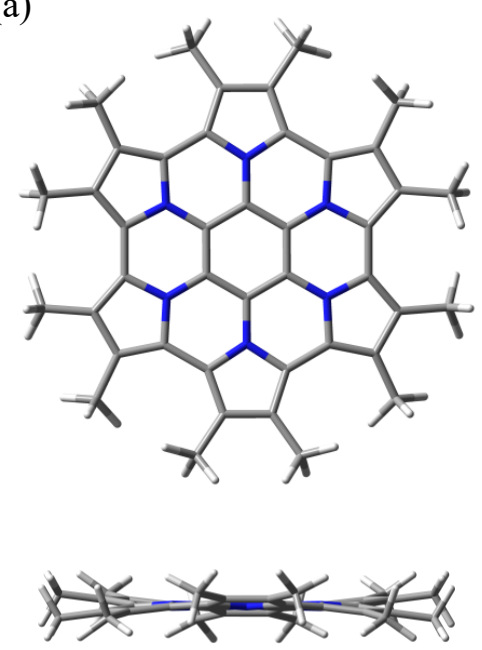

$C_{3 \mathrm{v}}$ symmetry

(d)
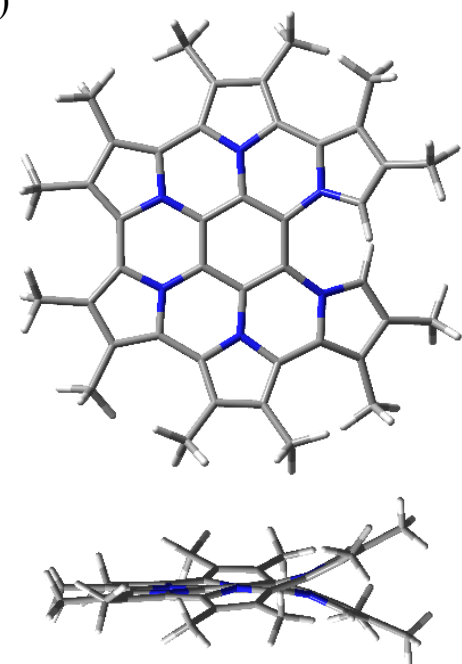

(b)
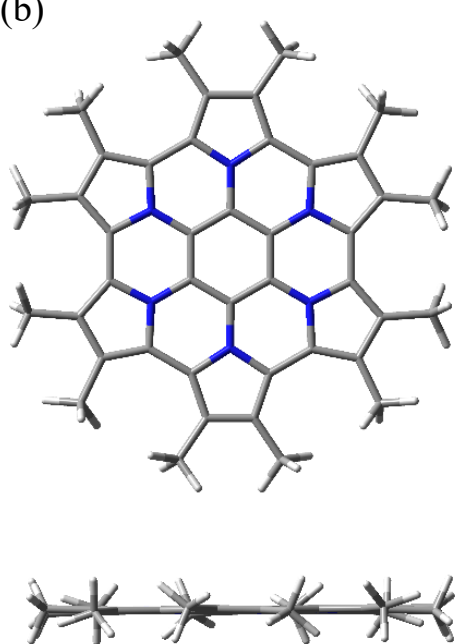

$C_{1}$ symmetry

(e)

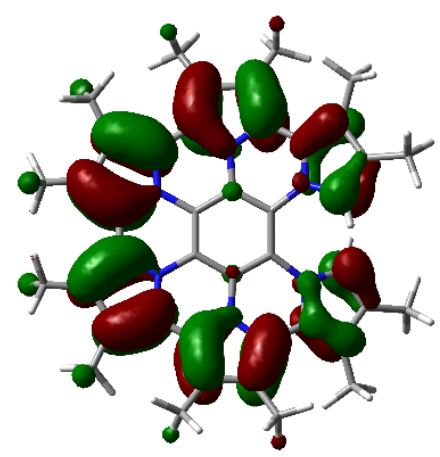

HOMO

$-4.06 \mathrm{eV}$ (c)
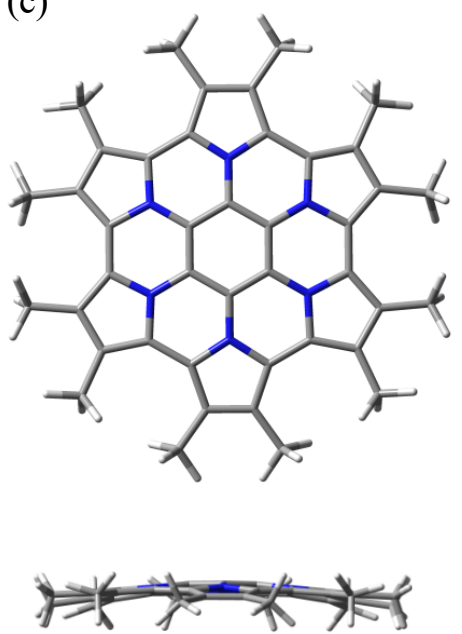

$C_{3 \mathrm{v}}$ symmetry

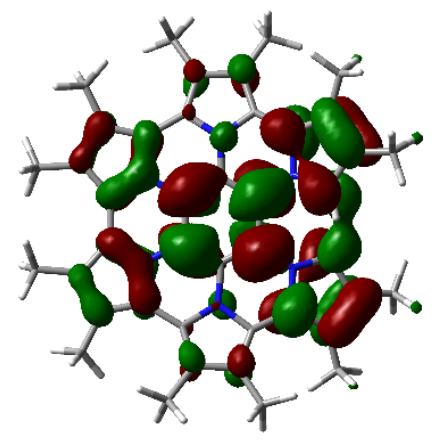

LUMO

$-0.78 \mathrm{eV}$

$C_{2}$ symmetry

Figure S7a. Optimized structures of (a) $\mathbf{2 d}$, (b) $\mathbf{2} \mathbf{d}^{++}$, (c) $\mathbf{2} \mathbf{d}^{2+}$, and (d) $\mathbf{3 d}$ from top and side view. (e) Frontier molecular orbitals of $\mathbf{3 d}$. 
Table S7. Bond lengths of $\mathbf{2 d}, \mathbf{2} \mathbf{d}^{\mathbf{}}$, and $\mathbf{2} \mathbf{d}^{2+}$.

\begin{tabular}{cccccc}
\hline & $C_{\alpha}-C_{\alpha}$ & $C_{\alpha}-C_{\beta}$ & $C_{\beta}-C_{\beta}$ & $N-C_{\alpha}$ & $C_{\text {Ben- }}-C_{\text {Ben }}$ \\
\hline $\mathbf{2 d}$ & 1.482 & 1.399 & 1.442 & 1.397 & 1.378 \\
$\mathbf{2 d}^{++}$ & 1.467 & 1.416 & 1.426 & 1.396 & 1.375 \\
$\mathbf{2 d}^{2+}$ & 1.449 & 1.434 & 1.412 & 1.395 & 1.374 \\
\hline
\end{tabular}

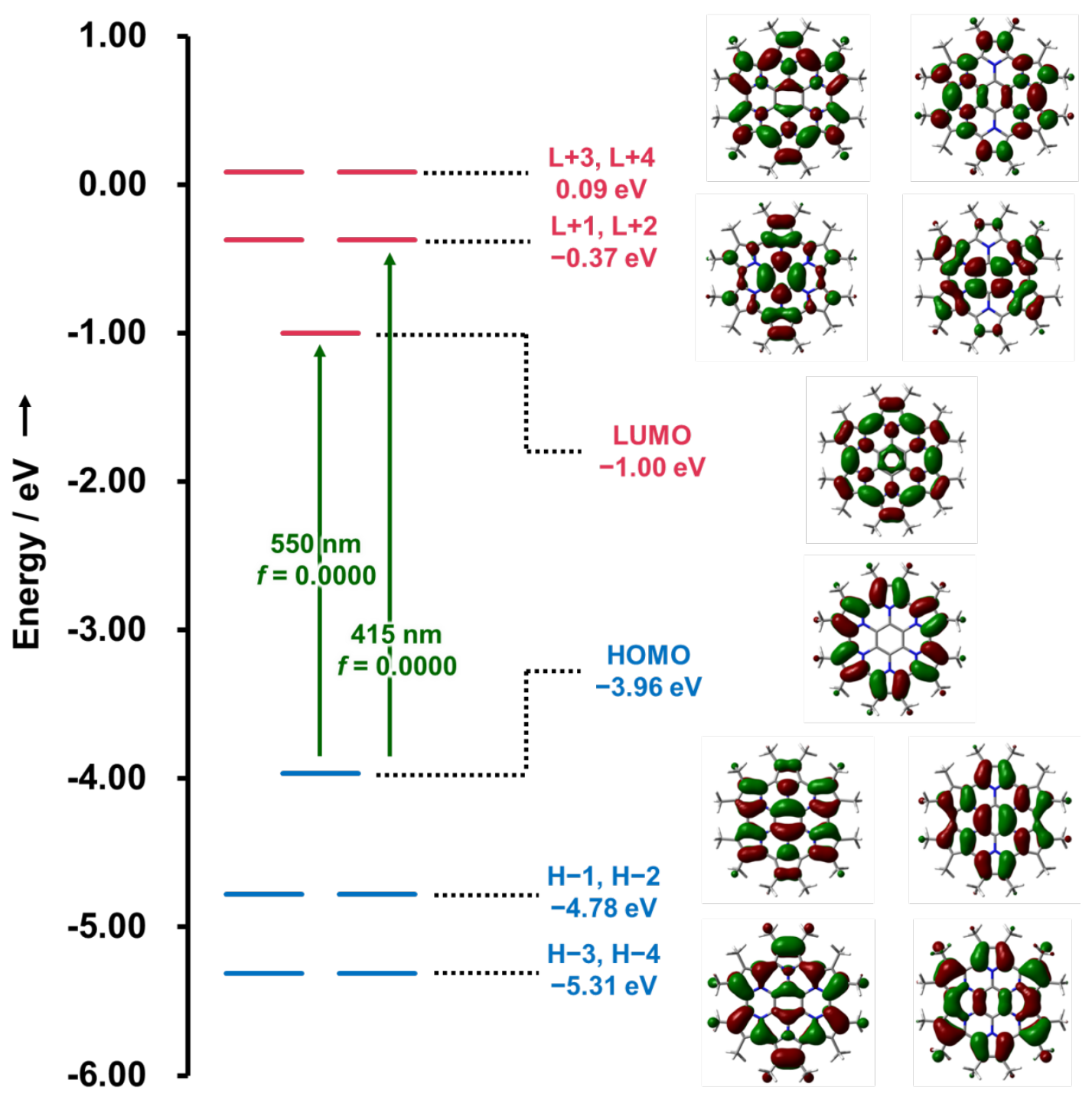

Figure S7b. Frontier molecular orbitals (isovalue $=0.02$ ) and orbital energies of $\mathbf{2 d}$. 


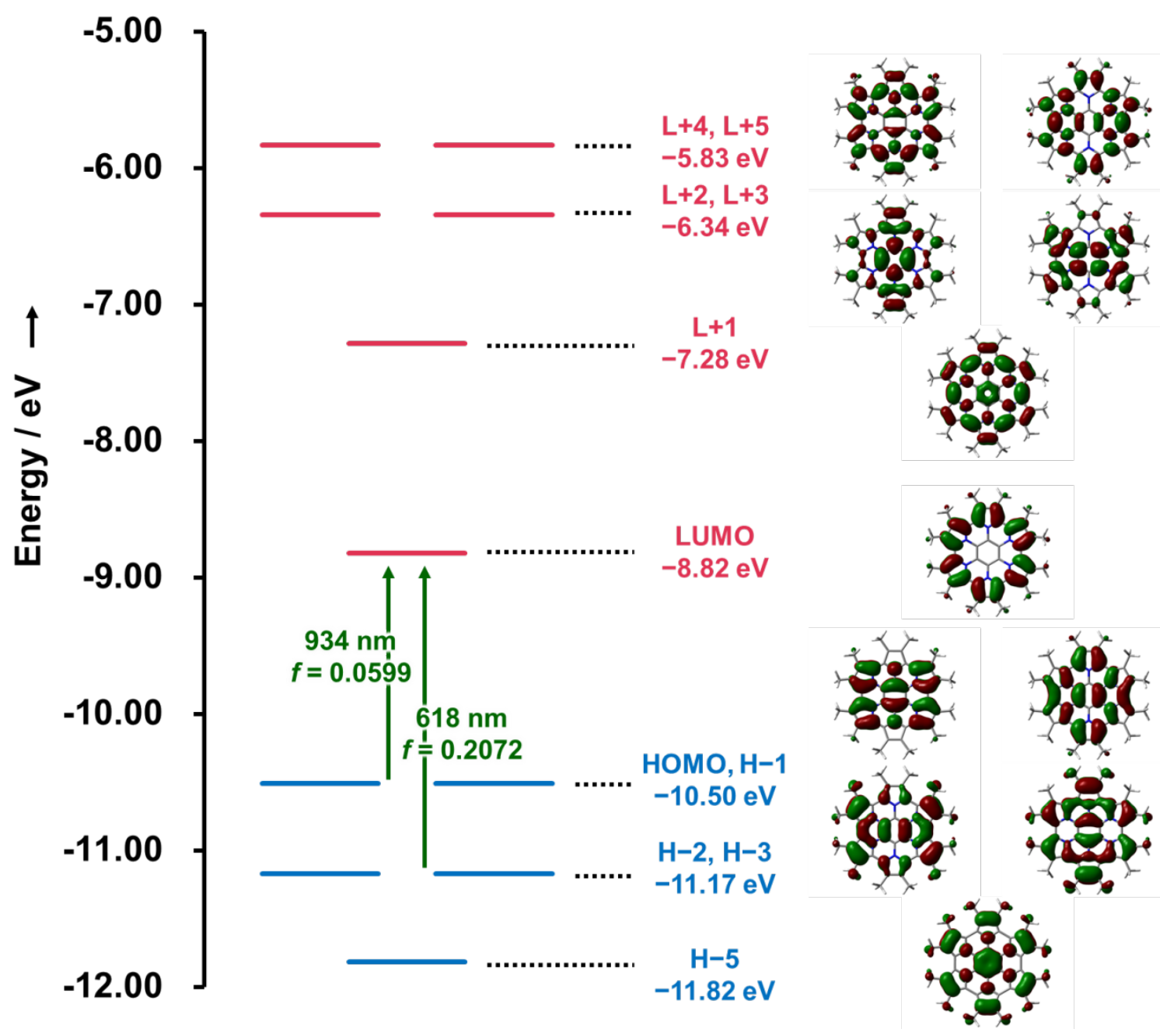

Figure S7c. Frontier molecular orbitals (isovalue $=0.02$ ) and orbital energies of $\mathbf{2} \mathbf{d}^{2+}$.

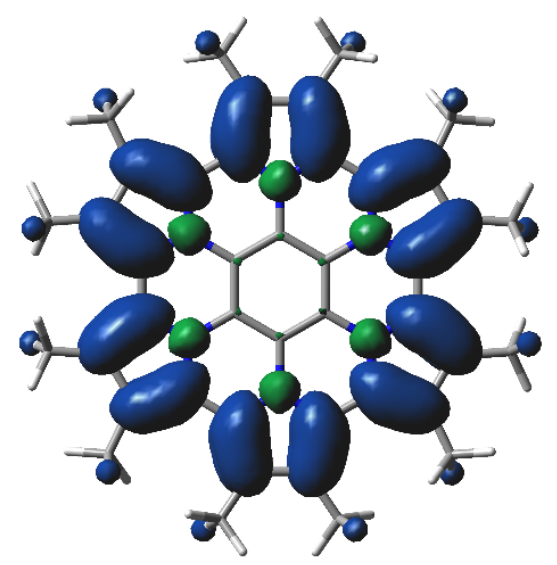

Figure S7d. Spin density map of $\mathbf{2 d}^{\mathbf{+}}($ isovalue $=0.004)$. 


\section{S8. Atomic coordinates of optimized structures}

Atomic coordinates of optimized structures calculated at the B3LYP/6-31G(d) level of theory were shown.

2d

C $\quad 1.19339054 \quad-0.68900435 \quad 0.01935420$

C $\quad-0.72096653 \quad-4.82000840 \quad 0.20071357$

C $\quad 0.00000000 \quad-1.37800348 \quad-0.01152568$

C $\quad-1.16909521 \quad-3.504613390 .03999993$

C $\quad-1.19339054-0.68900435 \quad 0.01935420$

C $\quad \begin{array}{llll}\text { C } & 1.56409031 & 6.04693843 & -0.39738879\end{array}$

C $\quad-1.19338602 \quad 0.68900174 \quad-0.01152568$

$\begin{array}{llll}\text { C } & -1.5609031 & 6.04693843 & -0.39738879\end{array}$

$\begin{array}{llll}\text { C } & -0.00000000 & 1.37800870 & 0.01935420\end{array}$

C $\quad 4.45475716 \quad-4.37801116 \quad-0.39738879$

$\begin{array}{llll}\text { C } & 1.19338602 & 0.68900174 & -0.01152568\end{array}$

$\begin{array}{llll}\text { C } & 4.4583644 & 4.37849984 & 0.39274621\end{array}$

$\begin{array}{llll}\mathrm{N} & 2.37631805 & -1.37196787 & 0.04078949\end{array}$

C $\quad-4.45475716 \quad-4.37801116 \quad-0.39738879$

$\begin{array}{llll}\mathrm{N} & 2.37616234 & 1.37187797 & -0.03635631\end{array}$

$\begin{array}{llll}\text { C } & -1.5697387 & -6.04811749 & 0.39274621\end{array}$

$\begin{array}{llll}\mathrm{N} & -0.00000000 & 2.74393574 & 0.04078949\end{array}$

$\begin{array}{llll}\text { C } & 1.56397387 & -6.04811749 & 0.39274621\end{array}$

$\mathrm{N} \quad-2.37616234$

$1.37187797 \quad-0.03635631$

$\begin{array}{llll}\text { C } & -4.4583644 & 4.37849984 & 0.39274621\end{array}$

$\begin{array}{llll}\mathrm{N} & -2.37631805 & -1.37196787 & 0.04078949\end{array}$

$\begin{array}{llll}\text { C } & 6.01981032 & 1.66961764 & 0.39274621\end{array}$

$\mathrm{N} \quad 0.00000000 \quad-2.74375593 \quad-0.03635631$

$\begin{array}{llll}\text { C } & -6.0884748 & -1.66892728 & -0.39738879\end{array}$

$\begin{array}{llll}\text { C } & 3.61959493 & -0.73985277 & -0.03793066\end{array}$

$\begin{array}{llll}\text { C } & 6.01884748 & -1.66892728 & -0.39738879\end{array}$

C $\quad 4.53434186 \quad-1.78539207 \quad-0.20184256$

$\begin{array}{llll}\text { C } & -6.01981032 & 1.66961764 & 0.39274621\end{array}$

C $\quad 3.81336582 \quad-3.03415920 \quad-0.20184256$

C $\quad 2.45052876 \quad-2.76473478 \quad-0.03793066$

$\begin{array}{llll}\text { C } & 2.45053662 & 2.76477285 & 0.03999993\end{array}$

$\begin{array}{llll}\text { C } & 3.81376645 & 3.03437953 & 0.20071357\end{array}$

$\begin{array}{llll}\text { C } & 4.53473298 & 1.78562887 & 0.20071357\end{array}$

$\begin{array}{llll}\text { C } & 3.61963183 & 0.73984055 & 0.03999993\end{array}$

$\begin{array}{llll}\text { C } & -1.16906618 & 3.50458754 & -0.03793066\end{array}$

$\begin{array}{llll}\text { C } & -0.72097603 & 4.81955128 & -0.20184256\end{array}$

C $\quad 0.72097603 \quad 4.81955128 \quad-0.20184256$

$\begin{array}{llll}\text { C } & 1.16906618 & 3.50458754 & -0.03793066\end{array}$

$\begin{array}{llll}\text { C } & -3.61963183 & 0.73984055 & 0.03999993\end{array}$

$\begin{array}{llll}\text { C } & -4.53473298 & 1.78562887 & 0.20071357\end{array}$

$\begin{array}{llll}\text { C } & -3.81376645 & 3.03437953 & 0.20071357\end{array}$

$\begin{array}{llll}\text { C } & -2.45053662 & 2.76477285 & 0.03999993\end{array}$

$\begin{array}{llll}\mathrm{H} & 2.51216534 & 5.80808533 & -0.88480868\end{array}$

$\begin{array}{llll}\mathrm{H} & 1.79591242 & 6.55796093 & 0.54753379\end{array}$

$\begin{array}{llll}\mathrm{H} & 1.05130461 & 6.77750091 & -1.03178364\end{array}$

$\begin{array}{llll}\mathrm{H} & -1.79591242 & 6.55796093 & 0.54753379\end{array}$

$\begin{array}{llll}\mathrm{H} & -2.51216534 & 5.80808533 & -0.88480868\end{array}$

$\begin{array}{llll}\mathrm{H} & -1.05130461 & 6.77750091 & -1.03178364\end{array}$

H $\quad 3.77386679 \quad-5.07964168 \quad-0.88480868$

$\begin{array}{llll}\mathrm{H} & 4.78140454 & -4.83428624 & 0.54753379\end{array}$

H $\quad 5.34383565 \quad-4.29920696 \quad-1.03178364$

$\begin{array}{llll}\mathrm{H} & 5.34646021 & 4.30047533 & 1.02506737\end{array}$

$\begin{array}{llll}\mathrm{H} & 3.77642708 & 5.08112118 & 0.88080596\end{array}$

$\mathrm{H} \quad 4.78036461 \quad 4.83335798 \quad-0.55360273$

$\begin{array}{llll}\mathrm{H} & -4.78140454 & -4.83428624 & 0.54753379\end{array}$

$\mathrm{H} \quad-3.77386679 \quad-5.07964168 \quad-0.88480868$

C $\quad-2.45052876 \quad-2.76473478 \quad-0.03793066$

C $\quad-3.81336582 \quad-3.03415920 \quad-0.20184256$

$\begin{array}{llll}\text { C } & -4.53434186 & -1.78539207 & -0.20184256\end{array}$

$\begin{array}{llll}\text { C } & -3.61959493 & -0.73985277 & -0.03793066\end{array}$

$\begin{array}{llll}\text { C } & 1.16909521 & -3.50461339 & 0.03999993\end{array}$

$\begin{array}{llll}\text { C } & 0.72096653 & -4.82000840 & 0.20071357\end{array}$

$\mathrm{H} \quad-5.34383565 \quad-4.29920696 \quad-1.03178364$

$\begin{array}{llll}\mathrm{H} & -2.51216648 & -5.81104238 & 0.88080596\end{array}$

$\begin{array}{llll}\mathrm{H} & -1.05109077 & -6.78040805 & 1.02506737\end{array}$

$\mathrm{H} \quad-1.79562849 \quad-6.55659618 \quad-0.55360273$

$\begin{array}{llll}\mathrm{H} & 1.05109077 & -6.78040805 & 1.02506737\end{array}$

$\begin{array}{llll}\mathrm{H} & 2.51216648 & -5.81104238 & 0.88080596\end{array}$ 


$\begin{array}{crrc}\mathrm{H} & 1.79562849 & -6.55659618 & -0.55360273 \\ \mathrm{H} & -3.77642708 & 5.08112118 & 0.88080596 \\ \mathrm{H} & -5.34646021 & 4.30047533 & 1.02506737 \\ \mathrm{H} & -4.78036461 & 4.83335798 & -0.55360273 \\ \mathrm{H} & 6.28859357 & 0.72992119 & 0.88080596 \\ \mathrm{H} & 6.39755101 & 2.47993270 & 1.02506737 \\ \mathrm{H} & 6.57599307 & 1.72323820 & -0.55360273 \\ \mathrm{H} & -6.57731696 & -1.72367468 & 0.54753379 \\ \mathrm{H} & -6.39514029 & -2.47829396 & -1.03178364\end{array}$

$\mathbf{2 d}^{++}$

$\begin{array}{lrrr}\mathrm{C} & -0.04513954 & 1.37393071 & 0.00468160 \\ \mathrm{C} & 1.16729714 & 0.72608177 & 0.00448653 \\ \mathrm{C} & 1.21242340 & -0.64784865 & 0.00471876 \\ \mathrm{C} & 0.04512663 & -1.37392119 & 0.00467169 \\ \mathrm{C} & -1.16730826 & -0.72607678 & 0.00464143 \\ \mathrm{C} & -1.21243658 & 0.64785233 & 0.00461899 \\ \mathrm{~N} & -0.08984979 & 2.73499788 & 0.00275397 \\ \mathrm{~N} & -2.41351754 & 1.28964566 & 0.00294017 \\ \mathrm{~N} & -2.32364565 & -1.44535228 & 0.00277698 \\ \mathrm{~N} & 0.08983503 & -2.73498967 & 0.00291001 \\ \mathrm{~N} & 2.41349243 & -1.28966224 & 0.00289957 \\ \mathrm{~N} & 2.32364352 & 1.44534807 & 0.00269312 \\ \mathrm{C} & -1.28523326 & 3.45504975 & -0.00462203 \\ \mathrm{C} & -0.87094987 & 4.80915230 & -0.01631653 \\ \mathrm{C} & 0.55348582 & 4.85596930 & 0.01306016 \\ \mathrm{C} & 1.05571110 & 3.53196262 & 0.00660560 \\ \mathrm{C} & -3.63479290 & 0.61445882 & -0.00407424 \\ \mathrm{C} & -4.60035250 & 1.65028641 & -0.01565621 \\ \mathrm{C} & -3.92865286 & 2.90729739 & 0.01323143 \\ \mathrm{C} & -2.53093318 & 2.68022124 & 0.00635964 \\ \mathrm{C} & -2.34957697 & -2.84061189 & -0.00377554 \\ \mathrm{C} & -3.72942980 & -3.15894036 & -0.01403820 \\ \mathrm{C} & -4.48218289 & -1.94870296 & 0.01502983 \\ \mathrm{C} & -3.58663123 & -0.85175531 & 0.00716931 \\ \mathrm{C} & 1.28520250 & -3.45503908 & -0.00455830\end{array}$

H $\quad-6.28603214 \quad-0.72844367 \quad-0.88480868$

$\begin{array}{llll}\mathrm{H} & 6.39514029 & -2.47829396 & -1.03178364\end{array}$

$\begin{array}{llll}\mathrm{H} & 6.57731696 & -1.72367468 & 0.54753379\end{array}$

$\mathrm{H} \quad 6.28603214 \quad-0.72844367 \quad-0.88480868$

$\begin{array}{llll}\mathrm{H} & -6.39755101 & 2.47993270 & 1.02506737\end{array}$

$\begin{array}{llll}\text { H } & -6.28859357 & 0.72992119 & 0.88080596\end{array}$

$\begin{array}{llll}\mathrm{H} & -6.57599307 & 1.72323820 & -0.55360273\end{array}$

Total energy: -1950.73777635 a.u.
C $\quad 0.87093062 \quad-4.80914981$
$-0.01628955$
C $\quad-0.55351263 \quad-4.85597839$
0.01291712
C $-1.05571813 \quad-3.53196229$
0.00657672
C $\quad 3.63477354 \quad-0.61448030$
$-0.00400849$
C $4.60032216 \quad-1.65030512$
$-0.01549433$
C $3.92862673-2.90732735$
0.01330898
C $\quad 2.53090472-2.68023285$
0.00643141
C 2.34958684
2.84060613
$-0.00389166$
C $\quad 3.72945981 \quad 3.15890707$
C $\quad 4.48218217 \quad 1.94866100$
$-0.01414827$
C $\quad 3.58662900$
0.85172746
0.01489206
C $\quad-5.98209051 \quad-1.88621182$
0.00708442
C $\quad-4.33659487 \quad-4.53183117$
0.06349546
C 1.35737190
6.12388059
$-0.06409746$
C -6.09286921
1.48991636
0.05953764
$\begin{array}{lll}\text { C } & 6.09284170 & -1.48986846\end{array}$
$-0.06696547$
C 5.98209956
1.88620114
$-0.06660251$
C 4.33675341
4.53173378
0.06313241
C -1.35751538
$-6.12383434$
$-0.06456272$
C -4.62479160
4.23741633
0.05901660
C $\quad 4.62481357 \quad-4.23743177$
0.05985282
C -1.75625309
6.02138001
0.06020385
C $\quad 1.75636687 \quad-6.02126614$
$-0.06817554$
H $\quad-6.42110527 \quad-1.68078239$
$-0.06834136$
H $\quad-6.32788747 \quad-1.10967607$
$-0.92123932$
0.74932471 


$\begin{array}{crrc}\mathrm{H} & -6.40248310 & -2.83132130 & 0.41409431 \\ \mathrm{H} & -4.34328833 & -5.01873224 & 0.91952857 \\ \mathrm{H} & -3.79513406 & -5.18379426 & -0.75315427 \\ \mathrm{H} & -5.37125766 & -4.49035045 & -0.41133168 \\ \mathrm{H} & 1.75443247 & 6.39980673 & -0.92578103 \\ \mathrm{H} & 2.20296257 & 6.03628736 & 0.74522694 \\ \mathrm{H} & 0.74914531 & 6.96100809 & 0.40903716 \\ \mathrm{H} & -6.51951573 & 1.25794433 & 0.91731653 \\ \mathrm{H} & -6.38594451 & 0.69133471 & -0.75202099 \\ \mathrm{H} & -6.57324198 & 2.40502239 & -0.42004491 \\ \mathrm{H} & 6.51926130 & -1.25750986 & 0.91767507 \\ \mathrm{H} & 6.38596869 & -0.69148801 & -0.75188675 \\ \mathrm{H} & 6.57339158 & -2.40503635 & -0.41926632 \\ \mathrm{H} & 6.32804088 & 1.10987578 & 0.74913405 \\ \mathrm{H} & 6.40252204 & 2.83142916 & 0.41337247 \\ \mathrm{H} & 6.42094355 & 1.68049083 & -0.92161318 \\ \mathrm{H} & 4.34413624 & 5.01868713 & 0.91903607 \\ \mathrm{H} & 3.79494849 & 5.18372760 & -0.75331661\end{array}$

$\mathbf{2 d}^{2+}$

$\begin{array}{llll}\text { C } & -1.19022057 & 0.68717417 & -0.35648885\end{array}$

$\begin{array}{llll}\text { C } & -1.18902224 & -0.68648231 & -0.34623954\end{array}$

$\begin{array}{llll}\text { C } & -0.00000000 & -1.37434833 & -0.35648885\end{array}$

$\begin{array}{lllll}\text { C } & 1.18902224 & -0.68648231 & -0.34623954\end{array}$

$\begin{array}{lllll}\text { C } & 1.19022057 & 0.68717417 & -0.35648885\end{array}$

$\begin{array}{lllll}\text { C } & 0.00000000 & 1.37296462 & -0.34623954\end{array}$

$\begin{array}{llll}\mathrm{N} & -2.36434100 & 1.36505292 & -0.22302910\end{array}$

$\begin{array}{lllll}\mathrm{N} & 0.00000000 & 2.72672539 & -0.20585668\end{array}$

$\begin{array}{llll}\mathrm{N} & 2.36434100 & 1.36505292 & -0.22302910\end{array}$

$\begin{array}{lllll}\mathrm{N} & 2.36141346 & -1.36336270 & -0.20585668\end{array}$

$\begin{array}{lllll}\mathrm{N} & -0.00000000 & -2.73010583 & -0.22302910\end{array}$

$\begin{array}{lllll}\mathrm{N} & -2.36141346 & -1.36336270 & -0.20585668\end{array}$

$\begin{array}{llll}\text { C } & -2.42406788 & 2.75128821 & -0.08046270\end{array}$

$\begin{array}{llll}\text { C } & -3.81616648 & 3.01835203 & 0.13905785\end{array}$

$\begin{array}{llll}\text { C } & -4.52205278 & 1.79572110 & 0.13905785\end{array}$

$\begin{array}{llll}\text { C } & -3.59471943 & 0.72366027 & -0.08046270\end{array}$

$\begin{array}{rrrc}\mathrm{H} & 5.37121189 & 4.49007539 & -0.41241347 \\ \mathrm{H} & -2.20262345 & -6.03668578 & 0.74540432 \\ \mathrm{H} & -0.74913707 & -6.96133068 & 0.40735663 \\ \mathrm{H} & -1.75535306 & -6.39890173 & -0.92622629 \\ \mathrm{H} & -4.12570476 & 4.92611775 & 0.74502135 \\ \mathrm{H} & -5.65356732 & 4.12918066 & 0.41020932 \\ \mathrm{H} & -4.66606039 & 4.71902594 & -0.92555031 \\ \mathrm{H} & 5.65326268 & -4.12914847 & 0.41148047 \\ \mathrm{H} & 4.66701473 & -4.71883631 & -0.92526452 \\ \mathrm{H} & 4.12519092 & -4.92631618 & 0.74479374 \\ \mathrm{H} & -1.20310369 & 6.89582859 & -0.41768091 \\ \mathrm{H} & -2.17377875 & 6.27269428 & 0.91525721 \\ \mathrm{H} & -2.59202062 & 5.87704930 & -0.75637766 \\ \mathrm{H} & 1.20350562 & -6.89559602 & -0.41859509 \\ \mathrm{H} & 2.17349167 & -6.27305089 & 0.91513841 \\ \mathrm{H} & 2.59240670 & -5.87644022 & -0.75609699 \\ & & & \\ \text { Total energy: }-1950.55523261 \text { a.u. } & \end{array}$

Total energy: -1950.55523261 a.u. 


\begin{tabular}{|c|c|c|c|c|c|c|c|}
\hline C & -3.59741762 & -0.72496262 & -0.10783601 & $\mathrm{H}$ & 1.92160619 & 6.24293049 & 1.08266481 \\
\hline $\mathrm{C}$ & -4.53691420 & -1.80392818 & -0.01150291 & $\mathrm{H}$ & -2.04759890 & -6.34273597 & -0.57936594 \\
\hline C & -3.83070473 & -3.02711887 & -0.01150291 & $\mathrm{H}$ & -2.35763230 & -5.82545219 & 1.08473724 \\
\hline $\mathrm{C}$ & -2.42654486 & -2.75297373 & -0.10783601 & $\mathrm{H}$ & -0.98859574 & -6.86642877 & 0.72204855 \\
\hline $\mathrm{C}$ & 4.42906234 & 4.36975732 & 0.35344408 & $\mathrm{H}$ & -3.96882472 & -5.09783528 & -0.59370134 \\
\hline $\mathrm{C}$ & 5.99885204 & 1.65080184 & 0.35344408 & $\mathrm{H}$ & -5.51529156 & -4.34214254 & -0.23980845 \\
\hline C & -5.99885204 & 1.65080184 & 0.35344408 & $\mathrm{H}$ & -4.44573331 & -4.78562502 & 1.08266481 \\
\hline $\mathrm{C}$ & 1.56155983 & 6.06014024 & 0.06275145 & $\mathrm{H}$ & -6.51805155 & -2.60531132 & -0.23980845 \\
\hline $\mathrm{C}$ & -1.56978968 & -6.02055915 & 0.35344408 & $\mathrm{H}$ & -6.39926723 & -0.88818539 & -0.59370134 \\
\hline $\mathrm{C}$ & -4.46745550 & -4.38242062 & 0.06275145 & $\mathrm{H}$ & -6.36733948 & -1.45730547 & 1.08266481 \\
\hline $\mathrm{C}$ & -6.02901535 & -1.67771965 & 0.06275145 & $\mathrm{H}$ & 6.39926723 & -0.88818539 & -0.59370134 \\
\hline $\mathrm{C}$ & 6.02901535 & -1.67771965 & 0.06275145 & $\mathrm{H}$ & 6.51805155 & -2.60531132 & -0.23980845 \\
\hline $\mathrm{C}$ & -1.56155983 & 6.06014024 & 0.06275145 & $\mathrm{H}$ & 6.36733948 & -1.45730547 & 1.08266481 \\
\hline $\mathrm{C}$ & 1.56978968 & -6.02055915 & 0.35344408 & $\mathrm{H}$ & -2.43044250 & 5.98602066 & -0.59370134 \\
\hline $\mathrm{C}$ & -4.42906234 & 4.36975732 & 0.35344408 & $\mathrm{H}$ & -1.00275997 & 6.94745385 & -0.23980845 \\
\hline $\mathrm{C}$ & 4.46745550 & -4.38242062 & 0.06275145 & $\mathrm{H}$ & -1.92160619 & 6.24293049 & 1.08266481 \\
\hline $\mathrm{H}$ & 3.86617345 & 4.95449556 & 1.08473724 & $\mathrm{H}$ & 2.04759890 & -6.34273597 & -0.57936594 \\
\hline $\mathrm{H}$ & 4.46917102 & 4.94464065 & -0.57936594 & $\mathrm{H}$ & 0.98859574 & -6.86642877 & 0.72204855 \\
\hline $\mathrm{H}$ & 5.45220390 & 4.28936341 & 0.72204855 & $\mathrm{H}$ & 2.35763230 & -5.82545219 & 1.08473724 \\
\hline $\mathrm{H}$ & 6.51676993 & 1.39809531 & -0.57936594 & $\mathrm{H}$ & -5.45220390 & 4.28936341 & 0.72204855 \\
\hline $\mathrm{H}$ & 6.22380576 & 0.87095664 & 1.08473724 & $\mathrm{H}$ & -4.46917102 & 4.94464065 & -0.57936594 \\
\hline $\mathrm{H}$ & 6.44079960 & 2.57706537 & 0.72204855 & $\mathrm{H}$ & -3.86617345 & 4.95449556 & 1.08473724 \\
\hline $\mathrm{H}$ & -6.22380576 & 0.87095664 & 1.08473724 & $\mathrm{H}$ & 5.51529156 & -4.34214254 & -0.23980845 \\
\hline $\mathrm{H}$ & -6.51676993 & 1.39809531 & -0.57936594 & $\mathrm{H}$ & 3.96882472 & -5.09783528 & -0.59370134 \\
\hline $\mathrm{H}$ & -6.44079960 & 2.57706537 & 0.72204855 & $\mathrm{H}$ & 4.44573331 & -4.78562502 & 1.08266481 \\
\hline $\mathrm{H}$ & 1.00275997 & 6.94745385 & -0.23980845 & & & & \\
\hline $\mathrm{H}$ & 2.43044250 & 5.98602066 & -0.59370134 & & 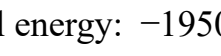 & 0.26766162 a.u & \\
\hline
\end{tabular}

\section{3d}

$\begin{array}{lrrc}\mathrm{C} & -0.01840669 & 0.68893954 & 1.08807747 \\ \mathrm{C} & 0.01840669 & -0.68893954 & 1.08807747 \\ \mathrm{C} & -0.04569295 & -1.37812915 & -0.11347236 \\ \mathrm{C} & -0.08399077 & -0.70707708 & -1.35391811 \\ \mathrm{C} & 0.08399077 & 0.70707708 & -1.35391811 \\ \mathrm{C} & 0.04569295 & 1.37812915 & -0.11347236 \\ \mathrm{~N} & -0.08220485 & 1.35603430 & 2.28772612 \\ \mathrm{~N} & 0.00067648 & 2.75999772 & -0.03413525\end{array}$




\begin{tabular}{|c|c|c|c|c|c|c|c|}
\hline C & -0.14688238 & 3.56332794 & -1.15703552 & $\mathrm{H}$ & -0.25142064 & 6.78409763 & -1.55291631 \\
\hline $\mathrm{C}$ & -0.58969424 & 4.80346191 & -0.70596526 & $\mathrm{H}$ & -1.87781655 & 6.50388907 & 1.01990163 \\
\hline $\mathrm{C}$ & -0.68180382 & 4.74596208 & 0.72480882 & $\mathrm{H}$ & -1.69875157 & 5.51484446 & 2.46652543 \\
\hline $\mathrm{C}$ & -0.30202961 & 3.46945827 & 1.13940188 & $\mathrm{H}$ & -0.36565934 & 6.53960361 & 1.91830744 \\
\hline $\mathrm{C}$ & 0.93561482 & 1.19102718 & -3.66505574 & $\mathrm{H}$ & 0.18110603 & 5.12447549 & 3.79802532 \\
\hline $\mathrm{C}$ & 1.19201147 & 2.33215865 & -4.38392242 & $\mathrm{H}$ & -1.26511933 & 4.68365143 & 4.71327609 \\
\hline $\mathrm{C}$ & 0.77283589 & 3.45080226 & -3.59446502 & $\mathrm{H}$ & 0.33581891 & 4.32994296 & 5.35960788 \\
\hline $\mathrm{C}$ & 0.24475170 & 2.95137657 & -2.40996472 & $\mathrm{H}$ & -0.73043454 & 2.54908091 & 6.39024413 \\
\hline $\mathrm{C}$ & -0.24475170 & -2.95137657 & -2.40996472 & $\mathrm{H}$ & -0.83907672 & 0.79733969 & 6.28802417 \\
\hline $\mathrm{C}$ & -0.77283589 & -3.45080226 & -3.59446502 & $\mathrm{H}$ & 0.73897024 & 1.57770669 & 6.43787362 \\
\hline $\mathrm{C}$ & -1.19201147 & -2.33215865 & -4.38392242 & $\mathrm{H}$ & 0.83907672 & -0.79733969 & 6.28802417 \\
\hline $\mathrm{C}$ & -0.93561482 & -1.19102718 & -3.66505574 & $\mathrm{H}$ & -0.73897024 & -1.57770669 & 6.43787362 \\
\hline $\mathrm{C}$ & 0.30202961 & -3.46945827 & 1.13940188 & $\mathrm{H}$ & 0.73043454 & -2.54908091 & 6.39024413 \\
\hline $\mathrm{C}$ & 0.68180382 & -4.74596208 & 0.72480882 & $\mathrm{H}$ & -0.33581891 & -4.32994296 & 5.35960788 \\
\hline $\mathrm{C}$ & 0.58969424 & -4.80346191 & -0.70596526 & $\mathrm{H}$ & -0.18110603 & -5.12447549 & 3.79802532 \\
\hline $\mathrm{C}$ & 0.14688238 & -3.56332794 & -1.15703552 & $\mathrm{H}$ & 1.26511933 & -4.68365143 & 4.71327609 \\
\hline $\mathrm{C}$ & 0.06594920 & -0.72794760 & 3.53801473 & $\mathrm{H}$ & 1.69875157 & -5.51484446 & 2.46652543 \\
\hline $\mathrm{C}$ & 0.17930735 & -1.76250527 & 4.47040748 & $\mathrm{H}$ & 0.36565934 & -6.53960361 & 1.91830744 \\
\hline $\mathrm{C}$ & 0.23674824 & -3.00937243 & 3.76389755 & $\mathrm{H}$ & 1.87781655 & -6.50388907 & 1.01990163 \\
\hline $\mathrm{C}$ & 0.21006526 & -2.73953378 & 2.39528937 & $\mathrm{H}$ & 1.92828772 & -6.42423271 & -1.15447732 \\
\hline $\mathrm{C}$ & -1.00185988 & 5.98216306 & -1.54019968 & $\mathrm{H}$ & 0.25142064 & -6.78409763 & -1.55291631 \\
\hline $\mathrm{C}$ & -1.17464007 & 5.87784936 & 1.57935065 & $\mathrm{H}$ & 1.19193782 & -5.68842777 & -2.57473983 \\
\hline $\mathrm{C}$ & -0.25404246 & 4.35313465 & 4.43693922 & $\mathrm{H}$ & -1.20392866 & -5.50490746 & -3.09311192 \\
\hline $\mathrm{C}$ & -0.25094893 & 1.66061367 & 5.96830301 & $\mathrm{H}$ & -1.86730239 & -4.96911570 & -4.63842412 \\
\hline $\mathrm{C}$ & 0.25094893 & -1.66061367 & 5.96830301 & $\mathrm{H}$ & -0.15021763 & -5.33357792 & -4.50182921 \\
\hline $\mathrm{C}$ & 0.25404246 & -4.35313465 & 4.43693922 & $\mathrm{H}$ & -2.75713508 & -2.98837859 & -5.72744502 \\
\hline $\mathrm{C}$ & 1.17464007 & -5.87784936 & 1.57935065 & $\mathrm{H}$ & -2.11047964 & -1.38172664 & -6.08087293 \\
\hline $\mathrm{C}$ & 1.00185988 & -5.98216306 & -1.54019968 & $\mathrm{H}$ & -1.17630595 & -2.82642598 & -6.49169698 \\
\hline $\mathrm{C}$ & -1.00185988 & -4.88564971 & -3.97160837 & $\mathrm{H}$ & 2.11047964 & 1.38172664 & -6.08087293 \\
\hline $\mathrm{C}$ & -1.83982999 & -2.38516526 & -5.73649845 & $\mathrm{H}$ & 1.17630595 & 2.82642598 & -6.49169698 \\
\hline $\mathrm{C}$ & 1.83982999 & 2.38516526 & -5.73649845 & $\mathrm{H}$ & 2.75713508 & 2.98837859 & -5.72744502 \\
\hline $\mathrm{C}$ & 1.00185988 & 4.88564971 & -3.97160837 & $\mathrm{H}$ & 1.86730239 & 4.96911570 & -4.63842412 \\
\hline $\mathrm{H}$ & 1.16554876 & 0.16453492 & -3.89657303 & $\mathrm{H}$ & 0.15021763 & 5.33357792 & -4.50182921 \\
\hline $\mathrm{H}$ & -1.16554876 & -0.16453492 & -3.89657303 & $\mathrm{H}$ & 1.20392866 & 5.50490746 & -3.09311192 \\
\hline $\mathrm{H}$ & -1.19193782 & 5.68842777 & -2.57473983 & & & & \\
\hline $\mathrm{H}$ & -1.92828772 & 6.42423271 & -1.15447732 & & ז & - & \\
\hline
\end{tabular}




\section{S9. TD DFT calculations}

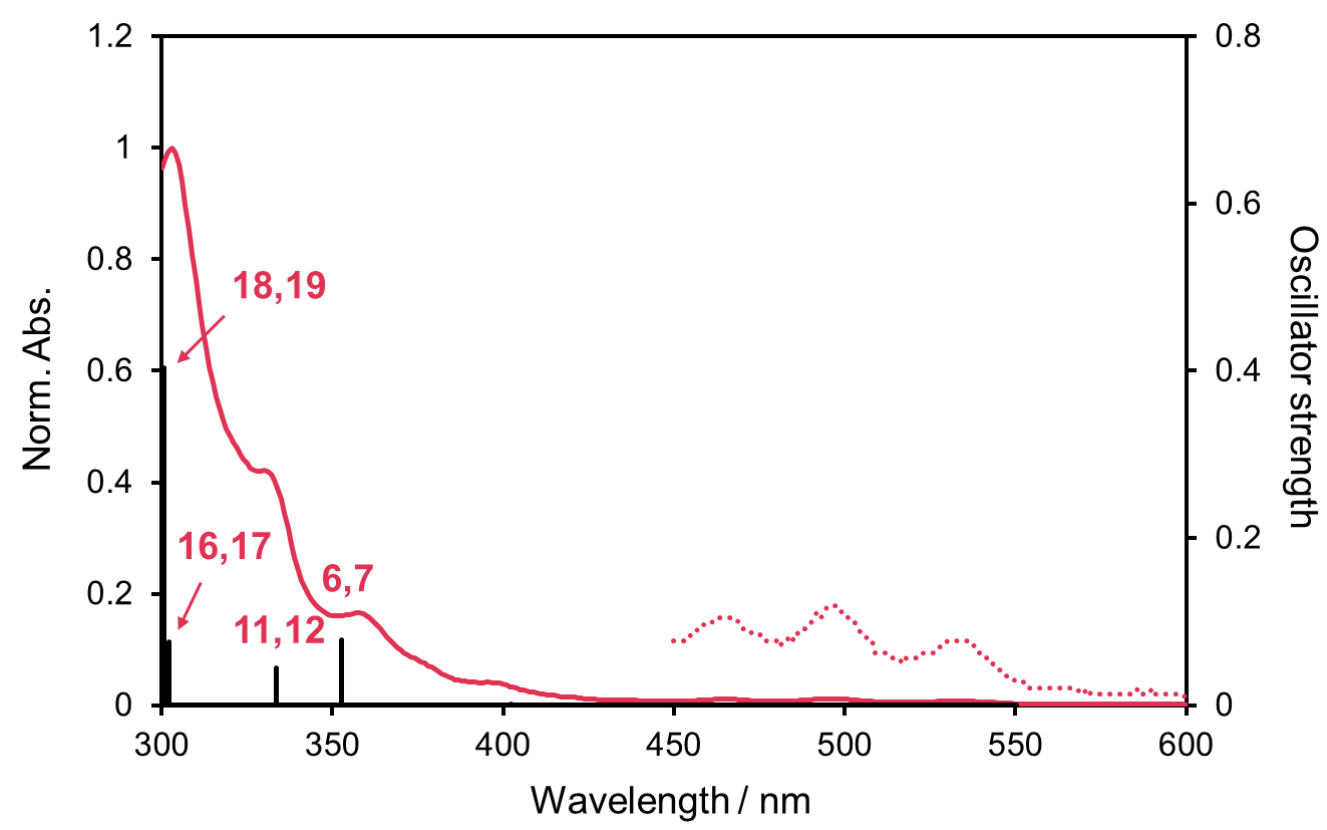

Figure S9a. Calculated stick spectra (B3LYP/6-31+G(d) level) of 2d along with the experimental absorption spectrum of $2 \mathrm{c}$ in $o$-dichlorobenzene.

Table S9a. Selected transition and oscillator strength of $2 \mathbf{d}$ calculated at the B3LYP/6-31+G(d) level of theory. Transitions whose compositions are larger than $c a .8 \%$ are shown.

\begin{tabular}{ccccc}
\hline $\begin{array}{c}\text { Exited } \\
\text { state }\end{array}$ & Transition & $\begin{array}{c}\text { Composition } \\
(\%)\end{array}$ & $\begin{array}{c}\text { Wavelength } \\
(\mathrm{nm})\end{array}$ & $\begin{array}{c}\text { Oscillator } \\
\text { Strength, } f\end{array}$ \\
\hline 1 & $\mathrm{H} \rightarrow \mathrm{L}$ & 99 & 550.2 & 0.0000 \\
2 & $\mathrm{H} \rightarrow \mathrm{L}+1$ & 99 & 415.4 & 0.0000 \\
3 & $\mathrm{H}-1 \rightarrow \mathrm{L}$ & 99 & 415.3 & 0.0000 \\
4 & $\mathrm{H}-2 \rightarrow \mathrm{L}$ & 91 & 402.3 & 0.0009 \\
5 & $\mathrm{H} \rightarrow \mathrm{L}+2$ & 91 & 402.3 & 0.0009 \\
6 & $\mathrm{H} \rightarrow \mathrm{L}+3$ & 82 & 352.6 & 0.0786 \\
7 & $\mathrm{H} \rightarrow \mathrm{L}+4$ & 82 & 352.6 & 0.0786 \\
& $\mathrm{H}-3 \rightarrow \mathrm{L}$ & 66 & & \\
11 & $\mathrm{H}-2 \rightarrow \mathrm{L}+2$ & 16 & 333.7 & 0.0445 \\
& $\mathrm{H}-1 \rightarrow \mathrm{L}+1$ & 16 & & \\
& $\mathrm{H}-4 \rightarrow \mathrm{L}$ & 66 & & \\
12 & $\mathrm{H}-2 \rightarrow \mathrm{L}+1$ & 16 & 333.7 & 0.0445 \\
& $\mathrm{H}-1 \rightarrow \mathrm{L}+2$ & 16 & & \\
\hline
\end{tabular}

\begin{tabular}{ccccc}
\hline $\begin{array}{c}\text { Exited } \\
\text { state }\end{array}$ & Transition & $\begin{array}{c}\text { Composition } \\
(\%)\end{array}$ & $\begin{array}{c}\text { Wavelength } \\
(\mathrm{nm})\end{array}$ & $\begin{array}{c}\text { Oscillator } \\
\text { Strength, } f\end{array}$ \\
\hline 16 & $\mathrm{H} \rightarrow \mathrm{L}+9$ & 85 & 302.1 & 0.0726 \\
17 & $\mathrm{H} \rightarrow \mathrm{L}+10$ & 84 & 302.1 & 0.0764 \\
& $\mathrm{H}-4 \rightarrow \mathrm{L}$ & 16 & & \\
& $\mathrm{H}-4 \rightarrow \mathrm{L}+2$ & 18 & & \\
18 & $\mathrm{H}-3 \rightarrow \mathrm{L}+1$ & 18 & 300.8 & 0.4032 \\
& $\mathrm{H}-2 \rightarrow \mathrm{L}+1$ & 15 & & \\
& $\mathrm{H}-1 \rightarrow \mathrm{L}+2$ & 15 & & \\
& $\mathrm{H} \rightarrow \mathrm{L}+9$ & 12 & & \\
& $\mathrm{H}-4 \rightarrow \mathrm{L}+1$ & 18 & & \\
& $\mathrm{H}-3 \rightarrow \mathrm{L}$ & 16 & & 0.3992 \\
19 & $\mathrm{H}-3 \rightarrow \mathrm{L}+2$ & 18 & & \\
& $\mathrm{H}-2 \rightarrow \mathrm{L}+2$ & 14 & 300.7 & \\
& $\mathrm{H}-1 \rightarrow \mathrm{L}+1$ & 14 & & \\
& $\mathrm{H} \rightarrow \mathrm{L}+10$ & 13 & & \\
\hline
\end{tabular}




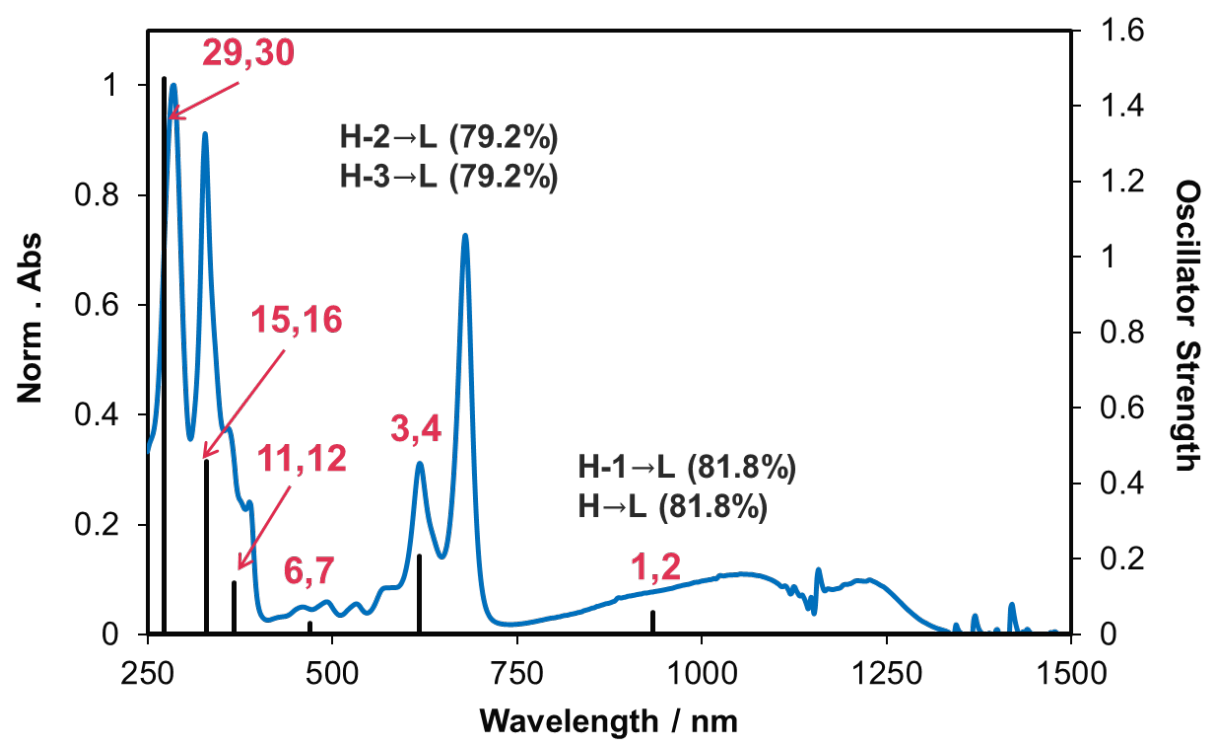

Figure S9b. Calculated stick spectra (B3LYP/6-31+G(d) level) of $\mathbf{2 d}^{2+}$ along with the experimental absorption spectrum of $\mathbf{2} \mathbf{c}^{2+}$ in $\mathrm{CH}_{2} \mathrm{Cl}_{2}$.

Table S9b. Selected transition and oscillator strength of $\mathbf{2 d}^{2+}$ calculated at the B3LYP/6-31+G(d) level of theory. Transitions whose compositions are larger than $c a .8 \%$ are shown.

\begin{tabular}{|c|c|c|c|c|}
\hline $\begin{array}{c}\text { Exited } \\
\text { state }\end{array}$ & Transition & $\begin{array}{c}\text { Composition } \\
(\%)\end{array}$ & $\begin{array}{c}\text { Wavelength } \\
(\mathrm{nm})\end{array}$ & $\begin{array}{l}\text { Oscillator } \\
\text { Strength, } f\end{array}$ \\
\hline \multirow{2}{*}{1} & $\mathrm{H}-2 \rightarrow \mathrm{L}$ & 16.9 & \multirow{2}{*}{933.5} & \multirow{2}{*}{0.0599} \\
\hline & $\mathrm{H}-1 \rightarrow \mathrm{L}$ & 81.8 & & \\
\hline \multirow{2}{*}{2} & $\mathrm{H}-3 \rightarrow \mathrm{L}$ & 16.9 & \multirow{2}{*}{933.5} & \multirow{2}{*}{0.0599} \\
\hline & $\mathrm{H} \rightarrow \mathrm{L}$ & 81.8 & & \\
\hline \multirow{2}{*}{3} & $\mathrm{H}-2 \rightarrow \mathrm{L}$ & 79.2 & \multirow{2}{*}{618.1} & \multirow{2}{*}{0.2072} \\
\hline & $\mathrm{H}-1 \rightarrow \mathrm{L}$ & 13.7 & & \\
\hline \multirow{2}{*}{4} & $\mathrm{H}-3 \rightarrow \mathrm{L}$ & 79.2 & \multirow{2}{*}{618.1} & \multirow{2}{*}{0.2072} \\
\hline & $\mathrm{H} \rightarrow \mathrm{L}$ & 13.7 & & \\
\hline 6 & $\mathrm{H}-1 \rightarrow \mathrm{L}+1$ & 88.7 & 469.7 & 0.0305 \\
\hline 7 & $\mathrm{H} \rightarrow \mathrm{L}+1$ & 88.7 & 469.7 & 0.0306 \\
\hline \multirow{3}{*}{11} & $\mathrm{H}-2 \rightarrow \mathrm{L}+1$ & 75.4 & \multirow{3}{*}{366.6} & \multirow{3}{*}{0.1377} \\
\hline & $\mathrm{H}-1 \rightarrow \mathrm{L}+2$ & 11.5 & & \\
\hline & $\mathrm{H} \rightarrow \mathrm{L}+3$ & 11.5 & & \\
\hline \multirow{3}{*}{12} & $\mathrm{H}-3 \rightarrow \mathrm{L}+1$ & 75.4 & \multirow{3}{*}{366.6} & \multirow{3}{*}{0.1377} \\
\hline & $\mathrm{H}-1 \rightarrow \mathrm{L}+3$ & 11.4 & & \\
\hline & $\mathrm{H}-1 \rightarrow \mathrm{L}+2$ & 11.5 & & \\
\hline
\end{tabular}

\begin{tabular}{|c|c|c|c|c|}
\hline $\begin{array}{l}\text { Exited } \\
\text { state }\end{array}$ & Transition & $\begin{array}{c}\text { Composition } \\
(\%)\end{array}$ & $\begin{array}{l}\text { Wavelength } \\
\text { (nm) }\end{array}$ & $\begin{array}{l}\text { Oscillator } \\
\text { Strength, } f\end{array}$ \\
\hline \multirow{5}{*}{15} & $\mathrm{H}-3 \rightarrow \mathrm{L}+1$ & 15.6 & \multirow{5}{*}{329.0} & \multirow{5}{*}{0.4596} \\
\hline & $\mathrm{H}-3 \rightarrow \mathrm{L}+2$ & 9.0 & & \\
\hline & $\mathrm{H}-2 \rightarrow \mathrm{L}+3$ & 8.9 & & \\
\hline & $\mathrm{H}-1 \rightarrow \mathrm{L}+3$ & 29.8 & & \\
\hline & $\mathrm{H} \rightarrow \mathrm{L}+2$ & 29.7 & & \\
\hline \multirow{5}{*}{16} & $\mathrm{H}-3 \rightarrow \mathrm{L}+3$ & 9.0 & \multirow{5}{*}{329.0} & \multirow{5}{*}{0.4597} \\
\hline & $\mathrm{H}-2 \rightarrow \mathrm{L}+1$ & 15.6 & & \\
\hline & $\mathrm{H}-2 \rightarrow \mathrm{L}+2$ & 9.0 & & \\
\hline & $\mathrm{H}-1 \rightarrow \mathrm{L}+2$ & 29.7 & & \\
\hline & $\mathrm{H} \rightarrow \mathrm{L}+3$ & 29.7 & & \\
\hline \multirow{2}{*}{29} & $\mathrm{H}-3 \rightarrow \mathrm{L}+2$ & 39.0 & \multirow{2}{*}{272.0} & \multirow{2}{*}{1.4724} \\
\hline & $\mathrm{H}-2 \rightarrow \mathrm{L}+3$ & 39.0 & & \\
\hline \multirow{2}{*}{30} & $\mathrm{H}-3 \rightarrow \mathrm{L}+3$ & 39.0 & \multirow{2}{*}{272.0} & \multirow{2}{*}{1.4726} \\
\hline & $\mathrm{H}-2 \rightarrow \mathrm{L}+2$ & 39.0 & & \\
\hline
\end{tabular}




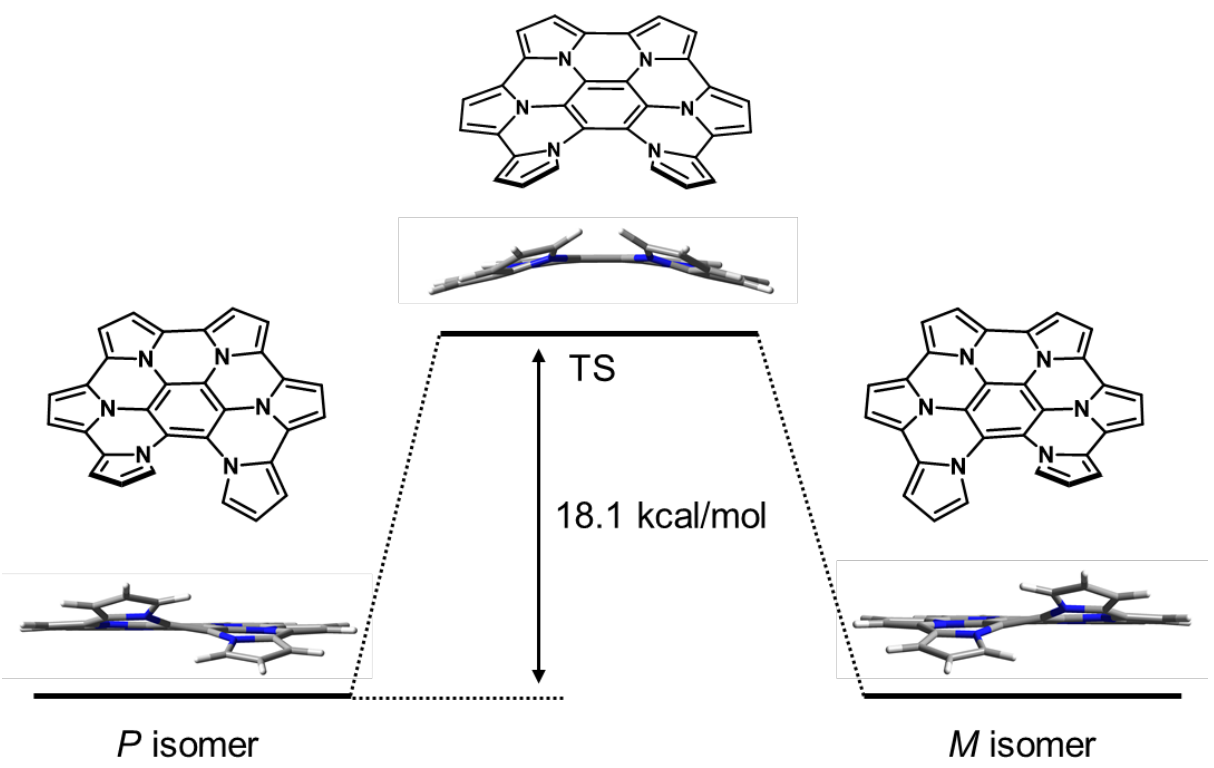

Figure S10. Inversion barrier of $\mathbf{3 c}$ calculated at the B3LYP/6-31G(d) level of theory. Ethyl groups are replaced with hydrogen atoms to simplify the calculation. 
S11. UV/vis/NIR absorption spectrum of $\left[\mathbf{2}^{-+}\right]\left[\mathrm{TCNQ}^{-}\right]$

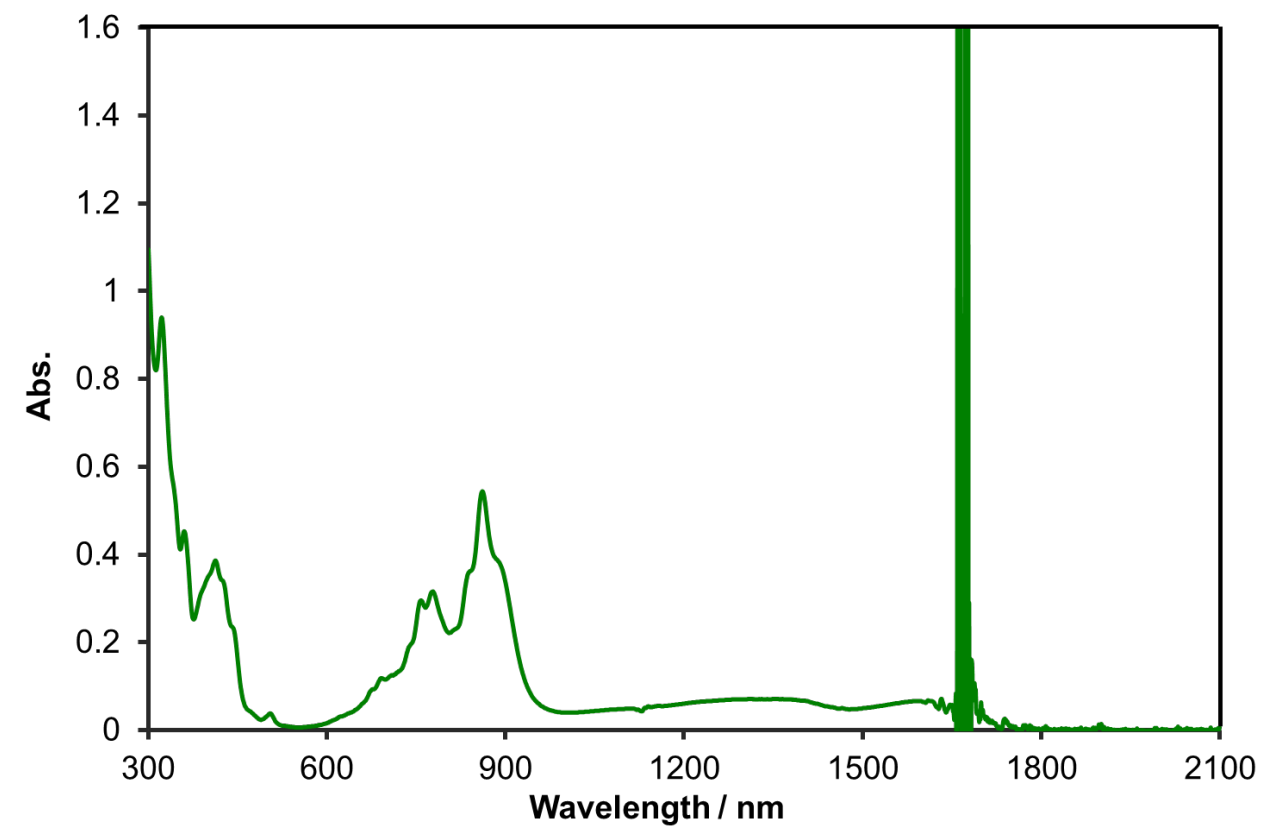

Figure S11. UV/vis/NIR absorption spectrum of $\left[\mathbf{1 c}^{\cdot+}\right]\left[\mathrm{TCNQ}^{-}\right]$in $o$-dichlorobenzene. 


\section{S12. References}

[1] SHELXT: G. M. Sheldrick, Acta Crystallogr. 2014, A70, C1437.

[2] Olex2: Dolomanov, O. V.; Bourhis, L. J.; Gildea, R. J.; Howard, J. A. K.; Puschmann, H. J. Appl. Cryst. 2009, 42, 339-341.

[3] SHELXL: G. M. Sheldrick, Acta Crystallogr. 2008, A78, 112.

[4] Gaussian 09, Revision D.01, Frisch, M. J.; Trucks, G. W.; Schlegel, H. B.; Scuseria, G. E.; Robb, M. A.; Cheeseman, J. R.; Scalmani, G.; Barone, V.; Mennucci, B.; Petersson, G. A.; Nakatsuji, H.; Caricato, M.; Li, X.; Hratchian, H. P.; Izmaylov, A. F.; Bloino, J.; Zheng, G.; Sonnenberg, J. L.; Hada, M.; Ehara, M.; Toyota, K.; Fukuda, R.; Hasegawa, J.; Ishida, M.; Nakajima, T.; Honda, Y.; Kitao, O.; Nakai, H.; Vreven, T.; Montgomery, Jr., J. A.; Peralta, J. E.; Ogliaro, F.; Bearpark, M.; Heyd, J. J.; Brothers, E.; Kudin, K. N.; Staroverov, V. N.; Keith, T.; Kobayashi, R.; Normand, J.; Raghavachari, K.; Rendell, A.; Burant, J. C.; Iyengar, S. S.; Tomasi, J.; Cossi, M.; Rega, N.; Millam, J. M.; Klene, M.; Knox, J. E.; Cross, J. B.; Bakken, V.; Adamo, C.; Jaramillo, J.; Gomperts, R.; Stratmann, R. E.; Yazyev, O.; Austin, A. J.; Cammi, R.; Pomelli, C.; Ochterski, J. W.; Martin, R. L.; Morokuma, K.; Zakrzewski, V. G.; Voth, G. A.; Salvador, P.; Dannenberg, J. J.; Dapprich, S.; Daniels, A. D.; Farkas, O.; Foresman, J. B.; Ortiz, J. V.; Cioslowski, J. and Fox, D. J. Gaussian, Inc., Wallingford CT, 2013. 